CAPÍTULO 7

\title{
COLABORACIÓN EN LA CADENA DE SUMINISTRO DE LÁCTEOS DEL DEPARTAMENTO DE SUCRE
}





\section{Introducción}

Desde la década de los 90's, las investigaciones han relacionado la colaboración en las cadenas de suministro como la estrategia de gestión de las relaciones internas y externas de las empresas que trabajan en sincronía por un objetivo común en el mediano y largo plazo, en la cual existe flujo de información, responsabilidades conjuntas, comunicación permanente, decisiones compartidas con el firme propósito de generar una ventaja competitiva a través del flujo continuo de operaciones y garantizar la satisfacción de los clientes. Esto se traduce en mayores beneficios en la cadena y que son compartidos de forma equitativa (Daugherty et al., 2006; Fawcett et al., 2008; Cao et al., 2010; Kumar et al., 2017; Zhang \& Cao, 2018). Dentro de los aspectos importantes de la colaboración, la confianza juega el papel principal al permitir que todos los miembros tengan acceso a la información, implicando de este modo también la responsabilidad de compartir los riesgos asociados (Tsanos, Zografos, \& Harrison, 2014; Wu \& Chiu, 2018).

La historia muestra la consolidación de evidencias que garantizan que la colaboración es una de las estrategias fundamentales para lograr una cadena de suministro eficiente, capaz de lograr una mejor calidad en sus productos, mejores prácticas operativas y reducción de los costos de tal forma que se tenga una respuesta oportuna y rentable a las necesidades cambiantes del mercado (Dania, Xing, \& Amer, 2018). Siendo vista desde el punto de vista de gestión contemplando aspectos de innovación, capacidad de la cadena y ventajas competitivas (Liao, Hu, \& Ding, 2017).

Por su parte, la integración de la cadena de suministro agroalimentaria comúnmente enfrenta desafíos significativos y complejos para lograr un desarrollo sostenible (Li, Wang, Chan , \& Manzini, 2014) incluidos aspectos económicos (Fluck, 2014), ambientales (Chounta, Hecking, Hoppe, $\&$ Avouris, 2014) y sociales que contemplan relación de dependencia 
a importaciones, gestión de residíos, bienestar animal, seguridad y trazabilidad de los alimentos, habilidades y satisfacción de trabajadores y reputación social (Gligor \& Holcomb, 2013).

La colaboración significativa entre partes interesadas heterogéneas es esencial para lograr la sostenibilidad en la cadena agroalimentaria. Una parte importante de las investigaciones en integración de la cadena se han centrado en examinar los factores que son críticos en la configuración de las características y la eficacia de la colaboración en la cadena de suministros. Sin embargo, existe una falta en el análisis estructurado sobre aspecto comunes de optimalidad como esfuerzos conjuntos, actividades de intercambio, valor de colaboración, adaptación, confianza, compromiso, poder, mejora continua, colaboración y estabilidad (Dania, Xing, \& Amer, 2018)

A pesar del creciente auge de visualización de la cadena de suministro colaborativa, pocas empresas se han visto beneficiadas plenamente de los recursos de información de sus socios en la cadena, revelando dos fuerzas esenciales en funcionamiento: el comportamiento de suministro de información, un método de apalancamiento de capital social y el comportamiento oportunista que mantiene cierto grado de asimetría de la información a fin de identificar antecedentes de visibilidad de los sistemas de información interorganizacional. (Lee, Kim, \& Kim, 2014).

Al hablar de colaboración en la cadena de suministro, es necesario contar con 7 componentes esenciales, dentro de los que se encuentra i) Integración: la cual permite asegurar que todas las transacciones interactúen a través de aplicaciones internas y externas, para el bien común de ganar clientes y aumentar la competitividad (Liu \&Yao, 2018), ii) Automatización y rediseños de los procesos: permitiendo el intercambio de información de manera automatizada y disponibilidad en tiempo real, iii) Tecnologías de la información: logrando el intercambio libre de datos, planes operativos e información financiera. (Han, Wang, \& Naim, 2017), iv) Confianza: al pensar que el socio actuará de acuerdo con un bien común". (Cho, Song, Comuzzi, \& Yoo, 2017), v) Dispersión geográfica: para garantizar la ágil respuesta, vi) Características del producto: para definir el ritmo de operación entre niveles y vii) Demanda del producto: a fin de identificar los cambios del mercado y adaptar la cadena. Lo anterior es necesarios en 
la aplicación de cualquiera de los tipos colaboración, descritos de acuerdo con la estructura definida en la Tabla 22.

Tabla 22. Clasificación de los tipos de colaboración en la cadena de suministro.

\begin{tabular}{|c|l|}
\hline $\begin{array}{c}\text { TIPO DE } \\
\text { COLABORACIÓN }\end{array}$ & \multicolumn{1}{c|}{ DESCRIPCIÓN } \\
\hline Compras colaborativas & $\begin{array}{l}\text { Permite la conexión con el proceso de planificación del } \\
\text { proveedor, mejorando las limitaciones de la materia } \\
\text { prima al proceso de producción/transformación. } \\
\text { (Miemczyk, Johnsen, \& Macquet, 2012). }\end{array}$ \\
\hline Estimación de Demandas & $\begin{array}{l}\text { Lograr la conexión y homogenización entre los diferentes } \\
\text { dominios de la planificación de la cadena identificando } \\
\text { los cambios dinámicos de la demanda y las } \\
\text { interrupciones en el suministro. (Moghaddam \& Nof, } \\
\text { colaborativas }\end{array}$ \\
\hline Inventarios & $\begin{array}{l}\text { Es una colaboración del enfoque de demanda, donde } \\
\text { existe flujo de información sobre el comportamiento del } \\
\text { mercado y niveles de inventario, encontrándose dentro } \\
\text { de esta la metodología de inventario administrado por el } \\
\text { proveedor (VMI) (Sana, 2012). }\end{array}$ \\
\hline Capacidad colaborativa & $\begin{array}{l}\text { Consiste en balancear las capacidades de las empresas en } \\
\text { colaboración a fin de mejorar la respuesta de cara al } \\
\text { proceso productivo y cliente. (Faust, Christens, Sparks, } \\
\& \text { Hilgendorf, 2015). }\end{array}$ \\
\hline Transporte colaborativo & $\begin{array}{l}\text { Permite organizar los transportes de acuerdo con la } \\
\text { agrupación táctica de productos y clientes. (Francois, } \\
\text { Moad, Bourrieres, \& Lebel, 2017). }\end{array}$ \\
\hline
\end{tabular}

Fuente: elaboración propia.

Esta clasificación permite definir la corriente temática de la investigación, al desarrollar un estudio de tipo descriptivo que logra caracterizar la cadena de suministros (SC) de los productos lácteos del departamento de Sucre, llevando el análisis desde los aspectos generales de colaboración, hasta la valoración de cada uno de los tipos de mencionados en la Tabla 22. Esto ayuda a conocer el estado actual de los componentes de la cadena y determinar el grado de integración existente entre cada eslabón. 
Para la implementación de las estrategias de colaboración las empresas cuentan con muchas opciones para formalizar la relación pudiendo ser en forma codificada, contractuales, informales y mutuas (Weber, Current, $\&$ Desai, 2000; Singh \& Power, 2009). Con la flexibilidad de aplicación entre fabricantes, intermediarios, proveedores y clientes siendo el caso más favorable en el que participan todos los componentes de la cadena, la integración con clientes permite el acceso a sus necesidades, segmentos de mercado, a la competencia, y otros productos y servicios. Mientras que la colaboración con los proveedores garantiza el abastecimiento de materiales evitando el efecto látigo (Holweg et al., 2005).

Dentro de los beneficios de la colaboración se relacionan el poder compartir riesgos, acceder a recursos complementarios, reducir los costos de transacción, retornos compartidos, compromisos, confianza, cultura colaborativa, mejorar de la productividad en el tiempo, el aumento de ventajas competitivas (Kalwani $\&$ Narayandas, 1995; Batt $\&$ Purchase, 2004; Bititci \& Mokadam, 2010; Kohli \& Jensen, 2010; Cao \& Vonderembse, 2010; Mathuramaytha, 2011; Cao \& Zhang, 2011; S. Tsanos et al., 2014; Narayanan et al., 2015; Liao et al., 2017; Kumar et al., 2017; Heirati \& Siahtiri, 2017), así como también mejoras en los ingresos, un impacto positivo en el rendimiento operativo para hacer frente a la incertidumbre de la demanda mejora la toma de decisiones (Fisher et al., 1997; Lee, Padmanabhan, \& Whang, 1997; Simatupang \& Sridharan, 2005; Mathuramaytha, 2011; Betts \& Tadisina, 2009), aumento en las ventas, mejora de los pronósticos e información más precisa y oportuna, reducción de los costos, de los inventarios y tiempos de entrega; mayores niveles de servicio, al aumentar la capacidad de respuesta al cliente para lograr su satisfacción (Bowersox, Closs, \& Stank, 2003; Kotabe, Martin, $\&$ Domoto, 2003; Betts \& Tadisina, 2009; Kohli \& Jensen, 2010), además del intercambio de conocimiento sobre productos y procesos; una mayor competitividad, oportunidad y una mejor proyección de la responsabilidad entre los socios, la eficiencia, eficacia y la posición en el mercado (Kotabe, Martin, \& Domoto, 2003; Kohli \& Jensen, 2010; S. Tsanos et al., 2014).

Sin embargo, para obtener estos beneficios, existen barreras operacionales y organizativas, haciendo referencia a la ausencia de integración interna, problemas de comportamientos, incompatibilidades tecnológicas entre otros por parte de las operacionales (Ramanathan, 2014) 
y la falta de confianza, el temor y otras situaciones internas y culturales que hacen parte de la incompatibilidad organizativa (Fliedner, G., 2003; Singh $\&$ Power, 2009; Cao \& Vonderembse, 2010).

La aplicación de las estrategias de colaboración puede ser una palanca poderosa en la cadena de suministro expuesta a condiciones de incertidumbre. Tal situación se evidencia en la cadena de suministros agroalimentarias, la cual, vista desde un punto socioeconómico es un sistema que agrupa actores económicos y sociales interrelacionados que participan articuladamente en actividades que agregan valor a un bien o servicio, siendo un círculo que va desde la planificación de la producción (toma de decisiones), la producción, la cosecha y llega hasta la distribución a los consumidores. Para La Gra, 2016, la incidencia que ejerce la incertidumbre en la cadena de suministros de agroalimentaria, está influenciada por factores inciertos como el clima, temperatura y preferencias del cliente (Jonkman et al., 2017), enfrentándose a grandes errores en la toma de decisiones traducido en altos costos de operación (Chen \& Li, 2008). Una forma de contrarrestar esta situación implica introducir los esquemas colaborativos, consiguiendo de esta manera una red de empresas comerciales de alimentos que integra proveedores de materiales e insumos, fabricantes, distribuidores, minoristas y consumidores que satisfacen la necesidad nutricional y de sustento humano (Zhang \& Li, 2012).

Los procesos principales involucrados en la cadena de suministros agroalimentaria abarcan aquellos relacionados al cultivo y cosecha de productos agrícolas (P1); almacenamiento y transporte de materias primas (P2); procesamiento y fabricación de materias primas (P3); almacenamiento y transporte de productos procesados y manufacturados (P4); distribución de bienes a los establecimientos al por mayor y al por menor (P5) y venta de bienes a los consumidores (P6), como se observa en la Figura 36. 


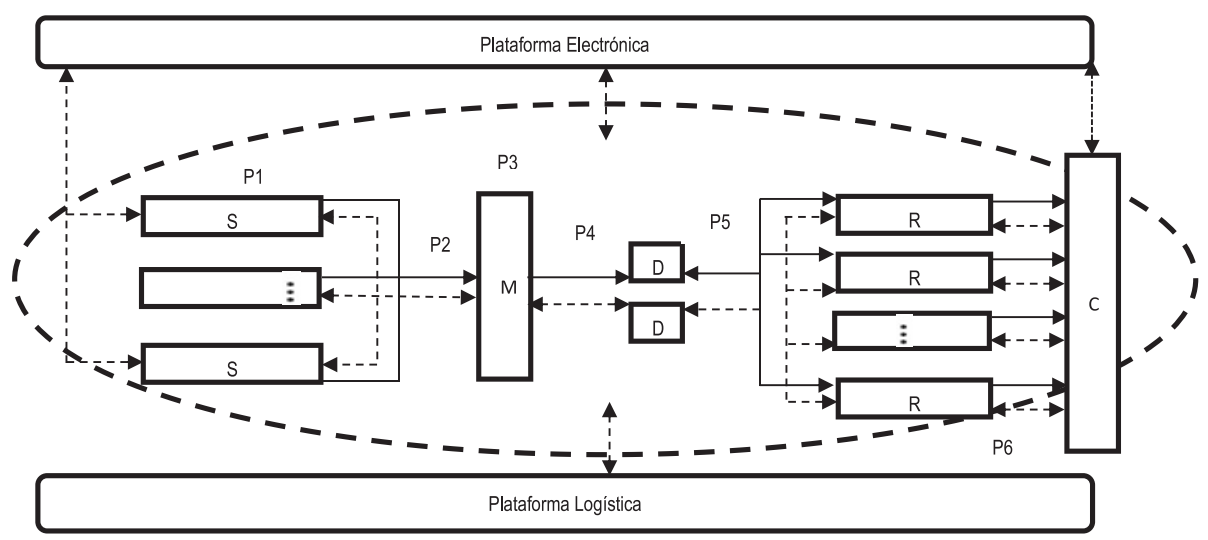

Figura 36. Procesos Involucrados en la Cadena de Suministros de Agro-productos. Fuente: Adaptación de Zhang \& Li (2012).

Del mismo modo, Jonkman, Bloemhof, van der Vorst, \& van der Padt, (2017) consideran la industria agroalimentaria, como parte de la industria alimentaria, relacionada con la conversión de los agro materiales en un conjunto de productos semi terminados y terminados que aportan a la nutrición de los seres humanos. En ello se ven involucradas etapas, actores y flujos como se muestra en la Figura 37. Este tipo de industria se distingue, por características como la estacionalidad, legislación y los cambios en la calidad del producto, lo que conduce a requisitos específicos para el transporte, almacenamiento y procesamiento (Jonkman et al., 2017).

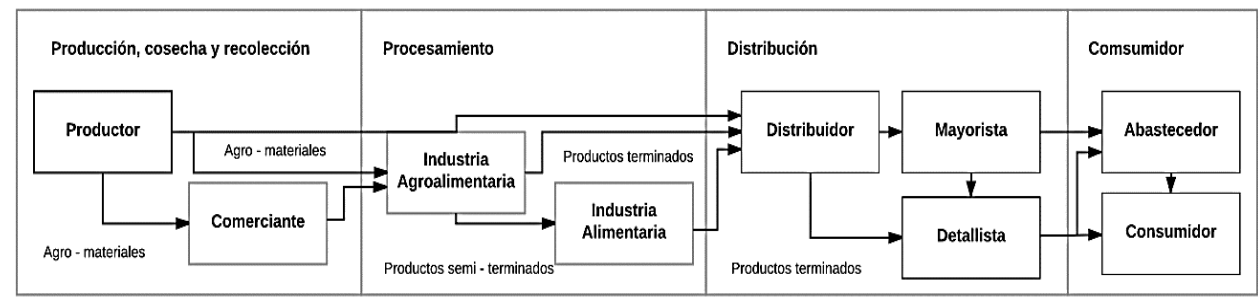

Figura 37. Representación de las Etapas y Actores Involucrados en una Cadena de Suministro de Alimentos, con sus Flujo Principal de Inventario .

Fuente: Adaptación Jonkman, et al. (2017).

Considerando la importancia de la estrategia de colaboración, tal y como lo plantea la literatura, se aborda la temática para evaluar el 
grado de aplicabilidad en el sector lácteo del departamento de Sucre, al ser una de las principales actividades económicas de la región, generando aproximadamente 24.610 litros de leche al año, representando el 2,6\% del total nacional (Proexport, 2013) con la participación de un inventario de 992.238 bovinos registrados en el censo de 2016. Cifras que captan el interés del estudio, permitiendo proporcionar un análisis de los componentes esenciales de operatividad de cada componente de la cadena, las principales problemáticas a los que se enfrentan los productores y los aspectos culturales que dificultan la optimización de las operaciones y el enfoque colaborativo. Los resultados obtenidos en este artículo hacen parte del estudio de caso particular aplicado en el desarrollo de modelos colaborativos que serán analizados y criticados en los futuros trabajos.

\section{Metodología}

La presente investigación es de tipo descriptiva desarrollada con el objetivo de caracterizar la colaboración en la cadena de suministro de productos lácteos del departamento de Sucre, permitiendo identificar las relaciones de las empresas del sector y la aplicación de estrategias colaborativas entre proveedores, fabricantes, distribuidores, clientes y demás miembros, contemplando aspectos como planificación, organización, procesos, investigación, desarrollo e innovación, incertidumbre ambiental y colaboración.

Para la recolección de la información se utilizaron encuestas desarrolladas en dos escenarios tal y como se muestra a continuación:

- Instrumento de Medición [1] enfoque de colaboración general.

- Instrumento de Medición [2] enfoque de colaboración de inventarios.

\section{Localización del Estudio}

El estudio se desarrolló en el departamento de Sucre considerando la aplicación del instrumento de medición [1] en la subregión Sabanas, en los municipios de Buenavista, Corozal, Galeras, Sincelejo, San Luis de Sincé, San Marcos, San Pedro, San Benito Abad y San Juan de Betulia y el instrumento de medición [2] en las subregiones Montes de María y Golfo de Morrosquillo en los municipios de Sincelejo, Ovejas, Morroa, Chalán, Coveñas, Palmito, San Onofre, Tolú y Toluviejo. 


\section{Determinación de la muestra}

La muestra se definió de acuerdo con la aplicabilidad de los instrumentos de medición en los municipios mencionados, considerando una población de 88 empresas registradas en Cámara de Comercio de Sincelejo, cuya actividad económica se encuentran relacionada con la elaboración de productos lácteos caso puntual aplicado en la producción de quesos en los municipios referenciados del departamento de Sucre y la cual es acotada al cruzarla con la base de datos de empresas con registro sanitario del Invima a 2017; se realizaron visitas y contacto vía telefónica de confirmación de funcionamiento, vigencia y actividad económica. Obteniendo de este modo un total de 21 empresas, las cuales al representar una cifra finita se convierte en la muestra de estudio.

\section{Resultados y análisis}

\section{Colaboración en la cadena de suministro de productos lácteos del departamento de Sucre}

\section{Escenario Mundial}

De acuerdo con las estadísticas de la de FAO, en los últimos 30 años, la producción mundial de leche ha aumentado en más del 30\%, pasando de 500 millones de toneladas en 1983 a 798,5 millones de toneladas en 2016. Para el año 2025, se prevé un aumento de 117 millones de toneladas, con una tasa de crecimiento promedio del 1,8\% por año, en los próximos 10 años. La distribución geográfica de la producción, para el 2016, se concentró en los países europeos, con el 32,7\%. Asía, produjo el 29,7\% y en el continente americano el 27,4\%. África y Oceanía, representó el 5,7\% y $4,5 \%$ respectivamente. El rendimiento a nivel mundial fue de 24.076 HG/AN (hectogramos por animal) para el año en mención según cifras de la FAO. (FAO, 2018).

A nivel mundial se ha buscado optimizar la producción de leche considerando la incertidumbre, con la finalidad de que se reducan los costos, incrementar estándares de calidad, mejorar sus componentes y rendimiento y ejecutar los procesos en forma sostenible. Logrando con esto satisfacer al cliente con productos confiables a un precio razonable. En el eslabón primario se ha analizado de la variabilidad en la producción de la leche en fincas, encontrando en el proceso de planeación los análisis 
de técnicas de predicción de la producción de leche diaria por rebaño realizando comparativos de diversas técnicas de predicción (Murphy, O'Mahony, Shalloo, French, \& Upton, 2014) y (Zhang, Murphy, Shallo, Ruelle, \& Upton, 2016). En el proceso de ejecución se ha prestado vital atención a la determinación de la raza (Shortall, Foley, Sleator, \& O’Brien, 2018) y los métodos e intervalos de ordeños aplicados a los animales (Perny, Crump, Hernandez, \& Reinemann, 2018).

La cadena de suministro de este sector, la integran productos primarios, proveedores de insumos, industrias transformadoras, empresas comercializadoras y distribuidoras, consumidores y agentes económicos interrelacionados, quienes añaden valor en el flujo del producto desde la producción primaria hasta el consumidor final. Los principales productos comercializados son leche esterilizada, leche pasteurizada, leche concentrada, leche UHT, leche en polvo, mantequilla, crema de leche, lactosuero, queso, yogurt y cuajada, (Ministerio de Agricultura y Desarrollo Rural, 2007).

\section{Escenario Nacional Colombiano}

El sector lácteo en Colombia es uno de los más importantes de su economía, representando el 2,3 de PIB nacional y el 24,3 del PIB agropecuario aportando una cuota de más de 700.000 empleos directos. Sin embargo, al hablar del sector lácteo es necesario referir la cadena de valor del sector ganadero, compuesta por dos sectores productivos (cárnicos y lácteo) los cuales contienen una gran cantidad de actores involucrados como: productores ganaderos, comercializadores, frigoríficos, industrias de transformación, distribuidores y el consumidor final. La organización de cada eslabón de la cadena se ha hecho en forma parcial a través de cooperativas entre productores e industria. (Lombana, y otros, 2012). 


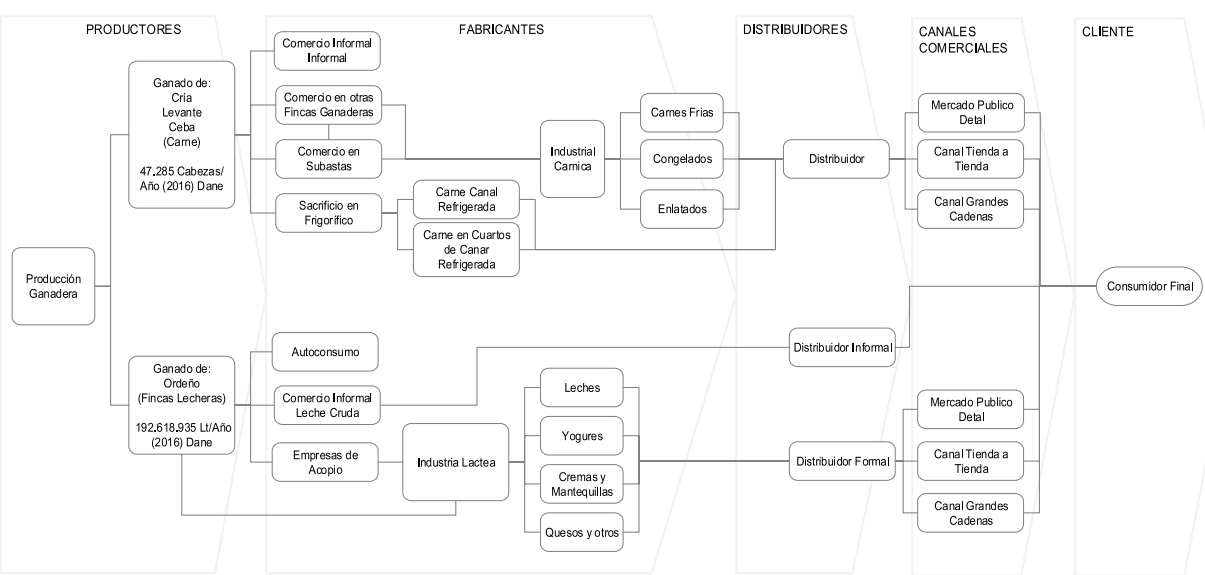

Figura 38. Estructura de La Cadena de Valor del Ganado.

Fuente: Adaptación de Lombana et. al., (2012).

La cadena de suministros del sector lácteo en el país se encuentra conformado por los siguientes eslabones, quienes permiten las relaciones e interacciones entre los ganaderos, acopiadores, industrias procesadoras, comercializadores, distribuidores y consumidor final (Fondo Nacional Ganadero - FNG, 2015) y los cuales se refieren a continuación:

\section{Producción primaria:}

Conformado por los hatos ganaderos, quienes se encargan de la producción de leche cruda bajo un sistema especializado o de doble propósito, según su ubicación geográfica (DANE, 2016). La productividad en este eslabón está representada por las regiones de Antioquia - Eje Cafetero, con un promedio de 15,5 litros/vaca/día, seguido de las regiones de Suroriente con 6,4, Santanderes-Otros con 5,9 y Costa Caribe con 3,4 litros/vaca/día (F.N.G., 2015). En el 2016, se registró un volumen de 6.391 millones de litros, distribuido en un 50,2\% para el acopio Industrial y de Cooperativas, $41,8 \%$ para ser procesado en finca o al consumo informal y un $8 \%$ al autoconsumo (F.N.G., 2018).

\section{Proveedores:}

Encargados de proveer insumos agropecuarios; de maquinaria y equipos agrícolas; servicios de veterinaria, asistencia técnica y equipos en 
general. Representa un eslabón transversal en toda la cadena de suministros (Calderòn \& Orjuela, 2004).

\section{Centros de Acopio:}

Son los encargados de la obtención de la leche y su almacenamiento temporal. Dentro de los componentes que lo integran se encuentra el intermediario, el cual se encargan de recolectar la leche en las fincas, transportarla y venderla a los procesadores, el transportador, el cual es contratado por el procesador para cumplir con dicho servicio y procesadores, quienes realizan el acopio directamente en las fincas (Consejo Nacional Lácteo - CNL, 2011).

\section{Plantas Productoras:}

Son empresas agroindustriales, encargadas de la transformación de la leche en una amplia familia de derivados lácteos, elaborados bajo los procesos de pasteurización, homogenización, evaporación, cadena de frio, entre otros. Según el Acuerdo de Competitividad de la Cadena Láctea Colombiana, 2010, un 88\% de la producción nacional de leche cruda es absorbida por los sub-eslabones dedicados a la pulverización y procesamiento de leche (CNL, 2010).

\section{Comercialización:}

Encargado realizar el cruce entre la oferta y la demanda, al llevar los productos terminados hasta el consumidor final. Esta función es realizada por intermediarios, que pueden ser mayoristas o minoristas representados en los canales de comercialización tradicional como tiendas de barrios y mercados públicos, y grandes superficies como los Supermercados de cadena y (Ministerio de Agricultura y Desarrollo Rural - MADR, 2007).

\section{Distribución:}

Conformado por las empresas o personas naturales encargados del transporte y la distribución de los productos, por medio de un transporte especializado o de manera informal (Orjuela, 2013).

Los datos del primer trimestre del año 2017 muestran que la economía colombiana registró un crecimiento representado en un PIB del 2\%. El sector agropecuario aporto al crecimiento con el 7,1 \% de los cuales el sector lácteo participó con una cuota del 9,6\% en producción de 
leche sin procesar. (DANE, 2018). Dentro de los aspectos evaluados para incrementar la competitividad del sector lácteo colombiano en el mercado nacional e internacional, se han concentrado el análisis de aspectos como la educación del personal involucrado en el proceso, reducción de costos de producción, gestión de reducción de precios de energía y combustibles, reducción de costos de impuestos, mejorar estructura y políticas crediticias, mejoras de la infraestructura vial, desarrollo de la asociatividad, entre otras (F.N.G, 2015).

Colombia no cuenta con la capacidad y tecnología en procesos para la exportación de derivados lácteos a países como Estados Unidos, debido a problemáticas sanitarias y la deficiente cadena de frio. El Tratado de Libre Comercio firmado con este país en el año 2012 se pactó un total de exportación de 6600 toneladas de queso y mantequilla por un plazo de desgravación de 11 años (Espinosa, 2012).

\section{Escenario Departamental Sucreño:}

En Sucre, las actividades económicas giran alrededor de la ganadería, la agricultura, el comercio y otros servicios. Gracias a las condiciones de los terreros para la actividad ganadera y la excelente calidad de su ganado vacuno, se cuenta con una magnífica cría, levante y ceba de animales de inmejorables condiciones para el consumo en los mercados regionales y la lechería, en menor escala (Aguilera-Díaz, 2005).

En el departamento la producción lechera es de tipo extensivo, con una escasa preparación tecnológica, un bajo nivel empresarial y productividad. La orientación ganadera está centrada en el doble propósito (65\%), seguido de cría con un 25\%; y ceba con el 10\%; el pequeño y mediano ganadero maneja su negocio de forma individual, y explota el campo de forma mixta, es decir combinando la agricultura con la ganadería hembras (Secretaria de Desarrollo Económico y Medio Ambiente, 2018).

En el 2016, el inventario bovino registra 326180 machos y 666058 hembras. Los municipios con mayor número de cabezas son San Onofre, San Marcos, Majagual, Sucre - Sucre, San Benito Abad, Santiago de Tolú, Sincé y Toluviejo. Para el 2017, se registró un total de 1.039.446 cabezas de ganado, donde el $67,7 \%$ corresponde a machos y el 32,3\% hembras. La mayor concentración (58\%) se sitúa en los municipios de San Luis de Sincé, San Marcos, San Onofre, Majagual, Sucre - Sucre, San Benito Abad, 
Galeras y Santiago de Tolú (Secretaria de Desarrollo Económico y Medio Ambiente, 2018).

Según las cifras del informe de coyuntura EVA, 2017, la ganadería sucreña produjo 217.390.533 litros de leche durante ese año, es decir una producción diaria de 595.591 litros y una producción en el hato de 2,875 litros por día en cada animal lechero. Los municipios con mayor producción corresponden a San Marcos, Majagual, Sincé, San Benito Abad, San Onofre, Galeras, Sucre - Sucre y Corozal. En el 2016, se registró una producción anual de 192.618.935 de litros de leche, equivalente a 527.723 litros diarios y una producción de 2,82 litros por día en cada animal lechero (Secretaria de Desarrollo Económico y Medio Ambiente, 2018).

De acuerdo con el sistema de explotación del ganado bovino, el mayor número de predios destinados corresponden al doble propósito en los municipios de San Marcos, Majagual, Sincé, San Benito Abad y San Onofre. Un total de 15000 granjas productoras se encuentran distribuidas para las explotaciones de carnes, leche y doble propósito en el departamento (Secretaria de Desarrollo Económico y Medio Ambiente, 2018).

El Caribe seco, en el cual se enmarca la ganadería de Sucre, presenta costos de producción competitivos a nivel nacional. El costo de producir un kilo de carne en pie es de $\$ 1.637$ y el de producir un litro de leche de $\$ 511$ aproximadamente, los cuales están por debajo del indicador nacional en $6.7 \%$ y $14 \%$, respectivamente. Por su parte el precio pagado al productor en promedio por cada litro de leche, si sitúa en \$ 882, para el 2016 y de $\$ 900$ en 2017. Los municipios con mayores precios fueron, Coveñas, San Pedro, San Antonio de Palmito, Buenavista y Sincelejo (Secretaria de Desarrollo Económico y Medio Ambiente, 2018).

En 2011, a través de una investigación se logró establecer las condiciones de comercialización de leche cruda para consumo humano directo en el municipio de Sincelejo - Sucre, encontrándose que la mayoría de los cruderos del municipio son hombres, productores o compradores de leche cruda, sin certificación como manipuladores de alimentos. El transporte se realiza en recipientes plásticos (51,7\%) y en la venta, un $72,6 \%$ se compra de manera directa y un 20,5\% se compra a intermediarios; en un rango de compra entre 20 a 400 litros/día por comercializador. La motocicleta, es el principal medio de transporte $(47,4 \%)$ y la comercialización se realiza 
al interior del municipio. Los tiempos prolongados en el transporte por las distancias entre los hatos lecheros y la baja cantidad de leche recorrida por kilómetros recorridos, representa un grave problema que repercute no solo en los tiempos y costos, sino en la calidad de la leche (Olivero, Aguas, \& Cury, Comercialización de Leche Cruda en Sincelejo, Sucre, Colombia, 2011).

De acuerdo con la investigación "Diagnóstico de la calidad composicional e higiénico sanitaria de la leche cruda bovina en hatos ganaderos del departamento de Sucre" desarrolla en 2009, los hatos productores de leche presentan una deficiencia en su infraestructura y solo el $74 \%$ de éstos poseen establos inadecuados; la obtención de la leche se realiza de forma manual solo en un 44,5\% y el resto combina sistemas mecánicos portátiles y fijos. La condición higiénica durante el ordeño es muy deficiente y no existe sistemas refrigerados para el almacenamiento de la leche cruda, además la mayoría de los hatos no cuentan con una fuente de agua potable ni poseen alcantarillado. En el departamento, según los resultados, todas las deficiencias en la obtención de leche afectan el proceso productivo y repercuten en la calidad final de la leche, siendo una leche de mala calidad higiénica, soportado por la prevalencia de elevados recuentos en microorganismos patógenos, por el contrario, se destaca como leche de excelente condición por su calidad bromatológica (Martínez, Serpa, \& Gómez, 2009).

La producción de leche en el departamento es de vital importancia, puesto que representa el sustento de una porción de la población, cuya actividad de venta permite satisfacer dos segmentos de mercado: aquellos clientes que consumen directamente el producto o utilizan la leche cruda para la elaboración de derivados lácteos. En Sucre, existe un total de 88 empresas agroindustriales inscritas hasta el 08 de septiembre de 2017 en la Cámara de Comercio de la ciudad, como empresas dedicas a la elaboración de productos lácteos.

Con relación a las empresas, la evaluación sobre el grado de competitividad de cinco empresas del sector lácteo en el municipio de Sincelejo, realizado en 2014, dio cuenta del bajo nivel en las empresas analizadas. Las áreas de producción, administración y talento humano son los componentes de mejor comportamiento, y contribuyen a mejorar el nivel de competitividad de estas (Hernández, Bustamante, \& Porto, 2014). 
En la misma línea de estudio, los autores Hernández, Vertel, \& Porto, 2016, evaluaron la incidencia de los componentes de producción, marketing, talento humano, procesos administrativos y finanzas en la competitividad de las empresas lácteas por medio de técnicas multivariadas exploratorias. El estudio realizado a cinco empresas agroindustriales lácteas demostró que existen solo dos empresas de mayor competitividad en la ciudad de Sincelejo (Hernández, Vertel, \& Porto, 2016).

Bajo este panorama y la necesidad de empezar a implementar herramientas que aumenten la capacidad de las empresas y los niveles de competitividad de la cadena de suministros del sector lácteo en el departamento de Sucre, es necesario analizar las relaciones entre los distintos actores de la cadena, los niveles de colaboración para así poder establecer las medidas y acciones que fortalezcan estas relaciones de colaboración y favorecer la productividad y competitividad de los actores.

\section{Caracterización de la colaboración en la cadena de suministro de lácteos del departamento de Sucre bajo enfoque generalizado}

Descripción eslabón industrial

Las empresas del sector lácteo del departamento de Sucre se caracterizan por ser microempresas de tipo familiar con una trayectoria entre los 5 y 10 años de funcionamiento; con una planta de personal menor a 10 personas en el $71 \%$ de la muestra. Las empresas son plantas de procesamiento, con líneas de producción de 1, 2 y 3 tipos de producto, donde predomina la producción de queso fresco tipo costeño, seguido del queso doble crema y queso tipo Mozarrella, como se observa en las Figuras 1 y 2 (Revisar enumeración respecto al documento). 
$\longrightarrow$ Series1

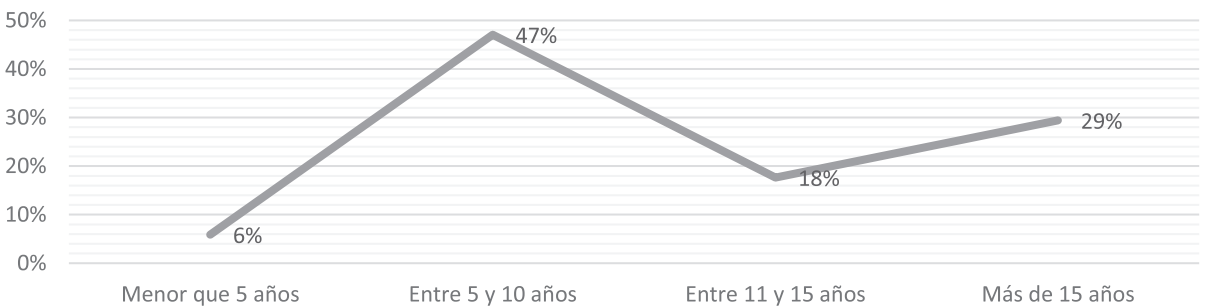

Figura 39. Tipos de Empresas en el sector lácteo del Departamento de Sucre.

Fuente: Adaptación de Lombana et. al., (2012).

Personal Familiar Sociedad Limitada Sociedad Anónima Otra

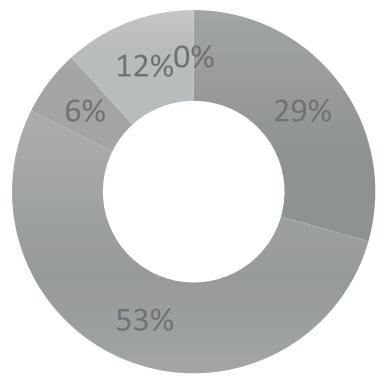

Figura 40. Antigüedad de las empresas en el Sector Lácteo del

Departamento de Sucre.

Fuente: elaboración propia.

Sobre su organización, presentan una planificación estratégica completa y organizada en el $94 \%$ de las empresas, mientras el 6\% se encuentra en procesos de construcción. Para las primeras, el 62\% utilizan los avisos y carteleras, un 10 \% charlas de divulgación; sin embargo, un 14\% no ha realizado divulgación de la misión, objetivos y organigrama dentro del personal. La estructura organizativa manejada en cada empresa responde a las estrategias de organización en un nivel alto el 41\%, medio (35\%), bajo (18\%) y nulo en un $6 \%$, en todas predomina un esquema de 
principios y valores. Los niveles de ejecución de las estrategias en cada organización se encuentran entre un 29\% y $41 \%$.

Con relación al área de mercadeo, que impulsa el crecimiento de las empresas, se encontró que el $71 \%$ de las empresas encuestadas del departamento no cuenta con un plan de mercadeo y un $41 \%$ argumenta un bajo interés en los procesos de investigación de mercado para las empresas. No obstante, por la trayectoria en el mercado, han identificado algunas debilidades para su crecimiento dentro de las que se citan: poca publicidad (71\%), precios que manejan (24\%) y canales de comercialización utilizados (18\%). En cuanto al aspecto de publicidad utilizado en el sector, se defiere en uso de publicidad impresa (45\%), el uso de las redes sociales (20\%), el contacto directo con clientes (20\%) y las páginas web (15\%) para impulsar sus productos.

En el segmento de mercado donde participan, las empresas han detectado una gama reducida de productos (39\%), precios altos de la competencia (28\%) y nuevos segmentos de mercados (20\%), como oportunidades para aprovechar. Sin embargo, el 71\% de las empresas nunca ha realizado alguna alianza estratégica con sus clientes para producir o comercializar el portafolio de sus productos.

Sobre el personal, en las empresas predomina un nivel medio de competencias laborales entre los empleados represen 49\%, bajo para un $29 \%$ de la planta y alto desempeño para otro $29 \%$. En cuanto al grado de formación educativa, predomina el secundario (63\%), un $15 \%$ es técnico y un 13 \% culminó la primaria. La percepción en relación con el empoderamiento del personal en la realización de sus funciones se encuentra entre medio y alto $(47 \% \mathrm{c} / \mathrm{u})$. Teniendo en las empresas encuestadas, un $53 \%$ argumenta que cuentan con convenios para capacitar al personal con entidades como Sena, Audilac S.A.S. Corposucre, entre otras. De este modo, se describe las motivaciones principales de desempeño de los colaboradores se encuentran el salario (32\%), la flexibilidad de los horarios de los turnos y el ambiente de trabajo ( $28 \% \mathrm{c} / \mathrm{u})$. Adicional, con una frecuencia media, se entregan incentivos dentro de la organización.

Con respecto a la parte productiva, de las empresas encuestadas, el $76 \%$ tiene manual de procesos y procedimientos, el 53\% maneja fichas técnicas de los productos y el 29\% flujogramas. Acerca de la infraestructura, 
el 88\% indica el uso exclusivo del local para actividades de la empresa y se encuentra separado del hogar, pero para el 12\%, este espacio forma parte del área de vivienda.

La cantidad de materia prima para la elaboración de derivados lácteos en las empresas supera los 7.000 litros de leche mensual y los 151 litros diarios. La mayoría de estas manejan almacenamientos propios para leche cruda. Un 56\% poseen un horario atípico de 7:00 a.m. a 11:00 a.m. para recibir los más de 40 proveedores manejados en tener cada empresa. Donde el mutuo acuerdo (59\%) predomina como la forma de pago entre los productores de leche y las empresas, seguido por la aplicación de la resolución 017 del 20 de enero del 2012 para fijar el precio y solo un 12\%, se rige por los precios del mercado. Utilizando métodos tradicionales en la comunicación de las necesidades de MP, realizado por vía telefónica (76\%) y solo un $18 \%$ utiliza documentos como pedidos, facturas o vales. A pesar de ello, todas las empresas manejan un tipo de control de entradas y salidas de materia prima de forma física (libro) en su área de recepción.

Para las pruebas de plataforma realizadas en el área de recibido, se encontró que, de las 17 empresas, el 47\% realiza análisis físico químico de la leche cruda, el 27\% solo análisis composicional y el 23\% verifica la calidad higiénico-sanitaria. Aquella materia prima no apta es devuelta al proveedor (44\%), y el 39\% la utiliza en la elaboración de derivados lácteos y un $17 \%$ de destina para la alimentación del ganado.

En la producción, esta se planifica de acuerdo con las condiciones ambientales de la zona (47\%), las cuales inciden en la disponibilidad de la leche cruda, un factor de planificación para un $26 \%$ de las plantas. Otras por su parte proyectan su producción según las necesidades del mercado (21\%). Los costos del proceso son calculados en un 94\% de las empresas encuestadas y un $6 \%$ además de calcularlos, las controlan. La planeación y control de la producción es muy importante para las empresas, por ello la estandarización de los procesos es alta (47\%) y en algunas medias (41\%).

A pesar de que la parte ambiental es importante para las empresas, solo un $41 \%$ de estas tiene un plan de residuos sólidos. Por otro lado, la calidad de los productos es controlada solo por un sistema rudimentario; pero, otras además poseen un control de calidad que se encuentra documentado. 
En cuanto a certificaciones, del total de empresas solo un $17 \%$ posee una línea de producción con HACCP y el 17\%, certificaciones en ISO 9001.

\section{Descripción eslabón transporte}

El transporte de la leche cruda en el departamento de Sucre se realiza a temperatura ambiente, indicando la carencia de una red de frio que garantice la calidad de la materia prima hasta su siguiente destino. En esta etapa se evidencia que los recipientes utilizados para el almacenamiento y transporte de la leche cruda corresponden en su mayoría a recipientes plásticos (65\%) y cantinas de acero inoxidable (35\%), siendo el plástico un material no permitido para esta actividad según el Decreto 616 de 2006 emitido por el Ministerio de Protección Social.

En cuanto al suministro del transporte, las empresas transformadoras, son las responsables de la movilización de la leche cruda producida en los hatos (68\%), hacia los centros de acopio o instalaciones de las plantas. En otras, lo realizan directamente los hatos productores o intermediarios. El tiempo empleado consume más de una hora y en vías destapadas (82\%). Teniendo como vehículo más utilizado de transporte, la motocicleta (435), seguido de la camioneta (29\%) y del camión de estaca (24\%). De estos vehículos, el $65 \%$ están en buen estado y tienen revisiones de tipo preventivo, el $24 \%$ son de manera correctiva y el $12 \%$ manifiesta que no tienen ningún mantenimiento.

Descripción relaciones entre actores de la cadena

Con relación a los productores de leche cruda, estos se encuentran conformados por hatos ganaderos dedicados a la explotación de carne y leche (71\%), hatos especializados (12\%), centros de acopios (12\%) y el sector informal (12\%). Las relaciones existentes entre productores y empresas trasformadoras, en un $71 \%$ están basadas en negociaciones para obtener materias primas a bajo costo, por otro lado, el 24\% argumenta que las relaciones se consolidan para tener poder de decisión entre varios proveedores. Los criterios de mayor importancia para selección de proveedores son la calidad de la leche cruda ofrecida (65\%) y las formas y plazos de pago (12\%) teniendo un $18 \%$ restante, el cual no realiza proceso de selección de proveedores.

Las entregas a tiempo de leche cruda y el número de devoluciones, se consolidan como los indicadores para medir el cumplimiento de los 
proveedores. Para las empresas una forma de contribuir a mejorar el rendimiento de sus proveedores, lo reflejan en el seguimiento a sus procesos (44\%), capacitaciones (6\%) y la participación en los pronósticos de la empresa (6\%). Sin embargo, un 44\% de las empresas indica que no aplica ninguna forma.

Con respecto a los clientes, todas las empresas tienen identificado su segmento de mercado y los comportamientos de compra de sus clientes, por ello solo ofrecen el portafolio de productos propios, los cuales frente a su competencia presentan una participación media (59\%), y alta para el $41 \%$ de las empresas. El nicho de mercado se caracteriza por excelentes productos y servicios (59\%), parámetros de valor agregado, otras con un calificativo bueno (41\%) y menores precios (21\%). La forma de conocer todos estos aspectos lo realizan por medio de retroalimentaciones directas de sus clientes (33\%), así mismo por las líneas de atención y correo electrónico (22\%) y un $17 \%$ por medio de las redes sociales. Sin embargo, en cuanto al entorno, un 35\% de las empresas trasformadoras del departamento, no realizan recolección de información alguna, un 29\% lo efectúa sobre los consumidores, clientes potenciales y características, un $24 \%$ sobre los productos de mercado y finalmente el $12 \%$ sobre el tamaño y características del mercado donde participa.

Las mayores ventas se concentran entre los meses de octubre y diciembre (47\%), seguido de los meses de julio a septiembre (21\%) y abril y junio (16\%); las condiciones ambientales de la región es un factor que afecta la disponibilidad de materia prima. Sin embargo, el 47\% de las empresas cuenta con suficiente capacidad para responder a la demanda, un porcentaje igual argumenta que muchas veces es insuficiente y un $6 \%$ con frecuencia no logran cubrir la demanda. Por ello, para un 48\% de las empresas, ampliar las instalaciones y comprar equipos (38\%), constituyen sus proyectos a mediano plazo.

Acerca del desarrollo de investigaciones en el sector, las empresas expresan que estas áreas no se contemplan dentro de la organización y la importancia de actividades en investigación, desarrollo o innovación es baja (41\%) a nula (29\%), no obstante, un 18\% indica que es medio y solo un $12 \%$, lo consideran alto. Para el desarrollo de nuevos productos o procedimientos, éstos no se cambian con frecuencia (53\%), y el $41 \%$ posee los mismos productos que su competencia. No obstante, una modificación 
del portafolio o servicio se genera cuando existe una solicitud de los clientes (78\%) o requerimientos de la gerencia (17\%) y solo participan personal de la empresa (89\%).

La participación en investigaciones con fines de trasferencia tecnológica es nula, así mismo como la ejecución de actividades de innovación o desarrollo (nulo a bajo). Esto también se refleja en los escasos convenios realizadlos con entidades públicas o privadas (65\%) y la nula participación de las empresas en planes de desarrollo del sector lácteo. Un panorama desalentador lo refleja la ausencia de asociatividad de las empresas, las cuales el 88\% argumenta no pertenecer a ninguna agremiación, asociación o cooperativa y solo el 29\% ha considerado establecer lazos de colaboración ya sea con los proveedores, clientes o empresas del mismo sector.

De acuerdo con los resultados generales obtenidos de la encuesta y con base en la revisión de fuentes secundarias y de la cadena láctea nacional, se puede establecer que, para el departamento de Sucre, la estructura que refleja la cadena de suministros en el sector lácteo se muestra en la Figura 41.

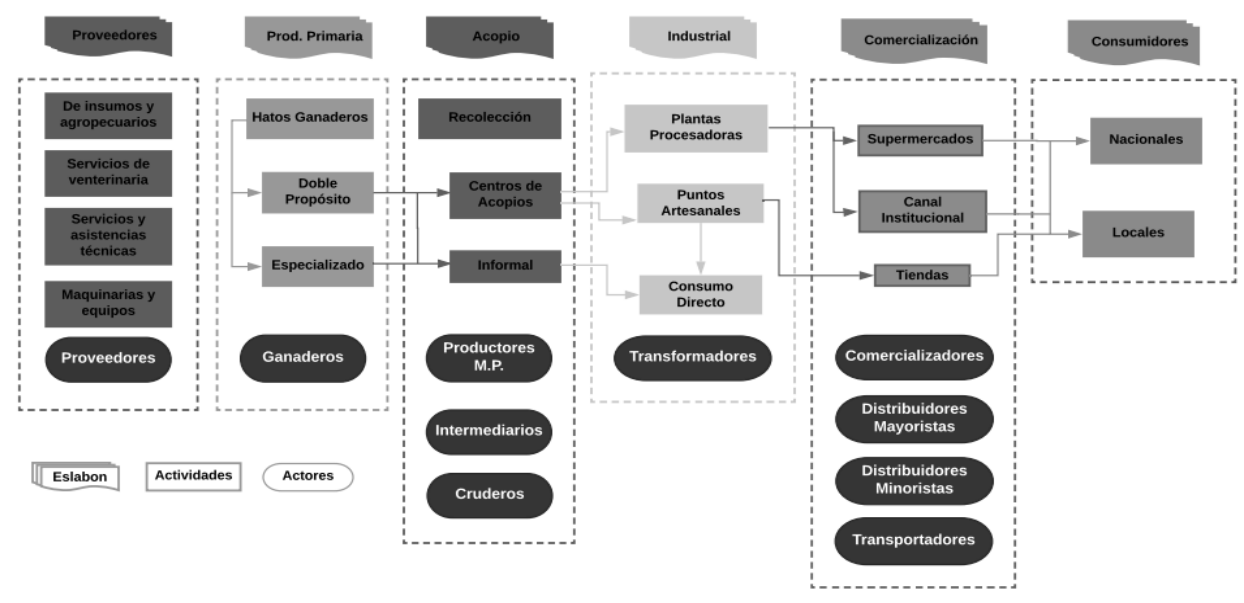

Figura 41. Cadena de suministros de lácteos del departamento de Sucre. Fuente: elaboración propia.

Un análisis en relación con la colaboración entre los actores de la cadena láctea del departamento nos muestra un esquema de actores independientes, quienes solo se encargan de llevar a cabo las etapas de 
producción, transporte, trasformación, comercialización y distribución, de acuerdo con las normativas vigentes e intereses propios. Por ello en la Figura 43 se propone un esquema de colaboración, que basado en alianzas y actividades colaborativas, intercambio de información, planificación conjunta, confianza y compromiso, permite articular los eslabones de cada actor en una interacción efectiva entre colaboradores, procesos, empresas, y entre sus actividades con el único fin de alcanzar metas y objetivos comunes para incrementar ventaja competitiva en la cadena láctea.

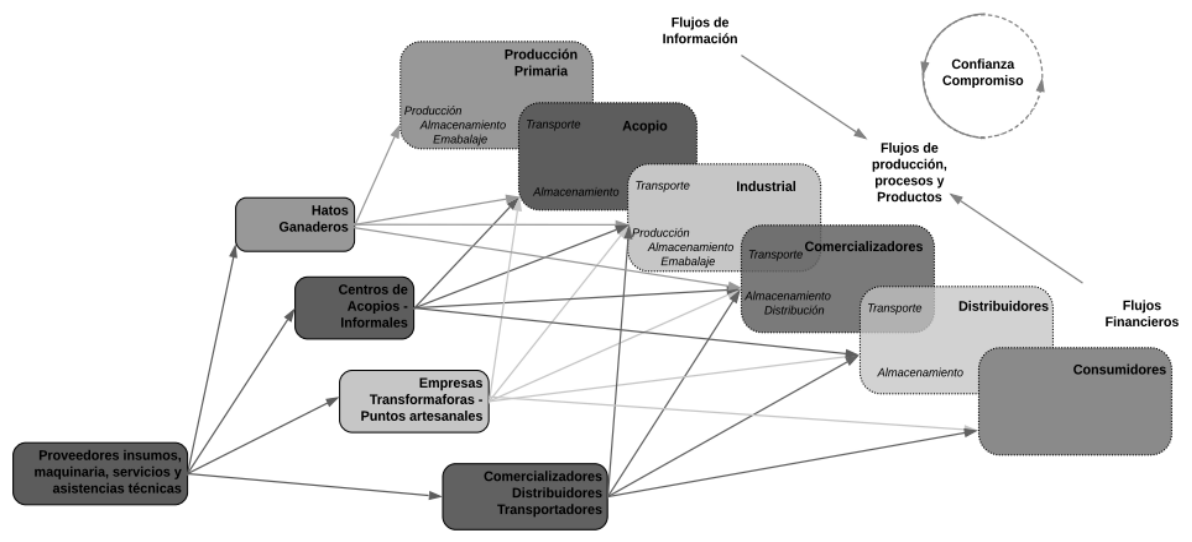

Figura 42. Esquema de Colaboración propuesto para la cadena de suministros de lácteos del Departamento de Sucre.

Fuente: Adaptación de Tsolaki, et al., (2014).

\section{Caracterización de la colaboración en la cadena de suministros del queso en el departamento de Sucre bajo enfoque de niveles de inventario.}

Las empresas de transformación de lácteos del departamento de Sucre se encuentran conformadas y dirigidas por personal capacitado, el cual fue clasificado de acuerdo con su máximo nivel educativo. Mostrando que el $57 \%$ son profesionales universitarios representando el mayor porcentaje, seguido por personal técnico con un 24\%, tecnólogo 10\% y bachiller y profesional especializado ambos con 5\%.

Los tipos de queso preferidos en la manufactura regional son el queso costeño y queso doble crema, siendo este último la base para la elaboración 
de otros tipos de quesos y productos. Cabe mencionar que el $62 \%$ de las empresas no es exclusiva de alguno de los dos tipos de quesos mencionado y que solo el 24\% de las plantas de transformación elaboran queso costeño, mientras que el 14\% solo elabora queso doble crema. Sin embargo, las empresas en búsqueda ampliar su portafolio y llegar a otros segmentos de mercado, alternan la producción con otros productos como suero costeño, leches procesadas, mantequilla, crema de leche, yogurt y otros. Lo que se traduce en sistemas de producción flexibles que se adaptan a los tipos de productos mencionados. El grado de participación de las empresas en cuanto a la elaboración de los productos mencionados, se refleja en la Figura 43.

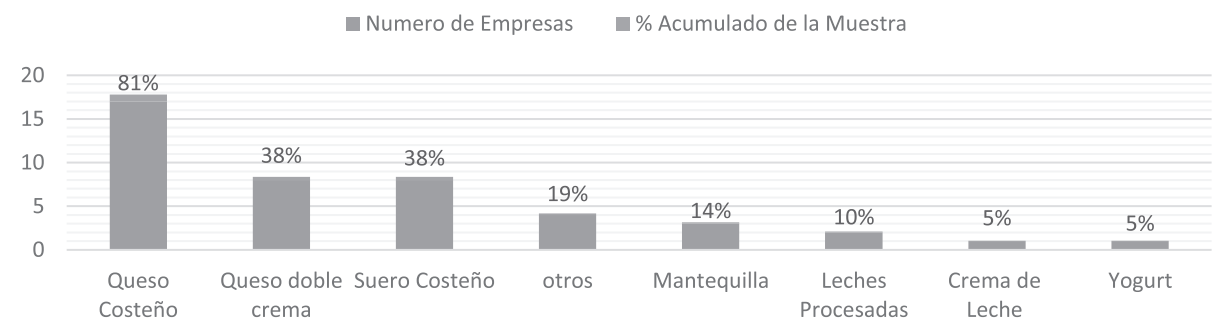

Figura 43. Clasificación de las empresas productoras según el tipo de producto elaborado.

Fuente: elaboración propia.

Planificación de la cadena de suministros

En cuanto a la planificación de operaciones de la cadena de suministro, se evidencia oportunidades estructurales, organizativas, culturales y de gestión que bien aplicadas podrían aportar grandes ventajas y desarrollo a la cadena. No obstante, la postura en cuanto al proceso de planificación en las empresas es percibida como un proceso de importancia pero que no alcanza estructuras solidas debido a la capacidad limita de personal y recursos, sumado a la desconfianza de los líderes de acceso de la información en las áreas internas y externas de la empresa. En general, la planificación de la demanda que permita gestionar los recursos óptimos del proceso productivo presenta un escenario critico que merece la pena intervenir, al encontrar que solo el 38\% de las empresas desarrolla algún tipo de planificación de la demanda el cual es transversal a todas las operaciones de la compañía, frente a un $62 \%$ que no refleja una planificación de la 
demanda en la que involucre a todas las etapas del proceso. Sin embargo, si realizan planificación de los materiales y recursos, un 90\% frente a un 10\% que manifiesta no hacerlo.

Por su parte las empresas en un 76\% realiza actividades de análisis de ventas el cual se presume es superficial al no contar con pronósticos o análisis estructurados para el óptimo desarrollo del proceso de producción mientras que el 24\% manifestaron no lo realizar análisis de ventas. Una de las limitantes que plantea la etapa y el sector es el sesgado acceso a la información, al ser las empresas manejadas en forma unipersonal considerando que a la hora de consultar y de planificar la producción se realiza como proceso multidisciplinario entre varias áreas de incidencia de las empresas a lo que se obtuvo que solo el $29 \%$ de las empresas lo realiza mientras que el $71 \%$ no lo hace.

De cara a medir la flexibilidad por devoluciones por parte de los miembros externos del proceso productivo en el proceso de planeación, se obtuvo que el $57 \%$ define rutas de recogida de los materiales, mientras que el $43 \%$ no participa las devoluciones en el proceso de planificación, dándose de forma espontánea.

En cuanto a la planificación general definidos por la empresa sea en forma estructurada o libre, se identifica que tal solo el $24 \%$ hace seguimiento al cumplimiento de las metas pactadas frente a un $74 \%$ que no tiene definido procedimientos y soportes de realizar seguimiento a los planes de acción definidos.

En la planificación de los canales de comunicación, las empresas no cuentan con sistemas de información que garanticen la información en tiempo real, esto debido a los costos asociados a la inversiones, la cual vista desde la perspectiva empresarial de Sucre presenta una tasa de retorno en el mediano y largo plazo, teniendo que solo el 38\% de las empresas encuestadas cuentan con sistemas de información definidos, frente a un $62 \%$ que no cuenta con sistemas que garanticen la información en tiempo real para la toma de decisiones. Sin embargo, el grado de efectividad de los sistemas utilizados reflejan la escasa inversión en tecnologías ya que, al medir en una escala de baja, intermedia, media, media alta y alta, se determina que el 5\% califica la efectividad de los sistemas como bajo, el $52 \%$ intermedio, el 24\% medio y el 19\% como media alta. 
Dentro de los aspectos importantes de un proceso productivo se encuentra la formación de la fuerza de trabajo, por lo que buscando medir la participación de las capacitaciones en el proceso de planificación se obtiene que el 62\% desarrolla cronogramas de capacitación en función de mejora de los procesos, mientras que el 38\% no las planifica y se dan en forma libre con restricción de tiempo. Lo que se evidencia en la frecuencia de realización, es que solo el 14\% realiza formación frecuentemente, frente a un $67 \%$ que las hace ocasional y un 19\% que las hace poco frecuentes.

Uno de los aspectos más críticos es la falta de control en los procesos y la poca intervención de estos aspectos en la etapa de planificación, al obtener que el $81 \%$ de las empresas no tiene estructurado indicadores que permitan medir el cumplimiento de los planes de acción definidos.

Abastecimiento de la cadena de suministros

El abastecimiento se enmarca en la relación existente con los proveedores en el suministro de productos o servicios por lo que este alcance se enfoca en la operatividad del abastecimiento. Es por ello que se parte del grado cumplimiento y solidez del proveedor a la hora de desarrollar el abastecimiento, se mide si las empresas productoras cuentan con algún sistema estructurado de evaluación para la selección de sus proveedores, obteniendo que solo el 19\% cuenta con una estructura definida y aplicada, mientras que el $81 \%$ refleja la informalidad de las negociaciones en la compra de materiales, insumos y servicios. Por lo que buscando profundizar en este aspecto se involucra el aspecto ambiental en la selección, observando que tan solo el 5\% de las empresas incluye aspectos ambientales en la evaluación y selección de sus proveedores frente a un $95 \%$ que no le da importancia a este aspecto.

Para el caso de la materia prima que ingresa al proceso de producción, se evidencia en la cadena que al no existir una planeación de los requerimientos de materiales MRP estructurado, el abastecimiento queda en función del criterio de las personas encargadas, representando este escenario el 52\% de las empresas encuestadas, frente a un 48\% que si efectúa su abastecimiento de acuerdo con el plan. Sin embargo, los MRP desarrollados no se complementan con pronósticos definidos de demanda lo que plantea un desbalance de la estructura de abastecimiento. En las 
empresas donde no se cuenta con MRP el abastecimiento en un 90\% se realiza a través de la revisión de la producción anterior.

Al igual que en la etapa de planificación son muchas las empresas que no definen indicadores de medición de la etapa de abastecimiento representando un $76 \%$ de éstas, quedando el control del proceso en la realización de inspecciones visuales y subjetiva que representan el 13\% de la muestra, quedando estas empresas sin instrumentos para la toma de decisiones. Esto trae consigo los escenarios con excesos de inventario de materiales e insumos o faltantes que se analizarán más adelante en otro de los alcances definidos. Sin embargo, las cantidades no son la única variable de decisión en el proceso de abastecimiento de queso y materiales, incluyendo el deterioro de éstos, los cuales son analizados y controlados en el $86 \%$ de las empresas.

Buscando medir la flexibilidad de los proveedores en el proceso de abastecimiento considerando cantidades y tiempo, se obtiene que el 14\% de las empresas lo califican como bajo, esto debido tal vez a políticas definidas por el proveedor, el $48 \%$ lo califica como media baja siendo el calificativo de mayor concentración, el 19\% lo califica como término medio y el 19\% restante lo califica como medio alto, reflejando la insatisfacción en el proceso y la necesidad de establecer acuerdos de colaboración en las empresas.

La operación de abastecimiento tiene asociado costos generalizados en el costo de abastecer, que puede tener penalidades por faltantes o excesos. Tener definido el costo implica tener control del proceso. Sin embargo, la escasa estructura de abastecimiento del sector lácteo del Departamento de Sucre solo presenta que el 19\% de las empresas tiene una estimación del costo. Por lo tanto, el control de este aspecto queda en función de otros costos analizados. La medición de su efectividad con relación al comportamiento real se encuentra en calificación dividida en 50\% y 50\% en la escala media y media alta mostrando un comportamiento estable para la toma de decisiones.

El abastecimiento en cuanto a funcionalidad interna de las empresas se encuentra representado en el proceso de compras y suministro, la cual debe dar respuesta oportuna a las necesidades de los otros procesos. De la métrica utilizada para medir el desempeño colaborativo del proceso de 
abastecimiento al interior de la empresa se obtiene que el comportamiento general es moderado con oportunidades, teniendo que el $5 \%$ de las empresas definió su colaboración interna como media baja, el 38\% la definió con un término medio, teniendo el porcentaje restante empresas dividido en partes iguales de $29 \%$ tenido valoraciones me media alta y alta respectivamente.

Con el fin de determinar la efectividad del proceso de abastecimiento y determinar la veracidad de la relación de integración entre procesos internos de las empresas, de evaluar la frecuencia de paradas de planta asociada a faltante de materiales, obteniendo cifras que posibilitan atribuir la causa a capacitación, falta de integración o simplemente a la falta de panificación de procesos en función de la demanda del mercado. Los resultados se presentan en la Figura 45.

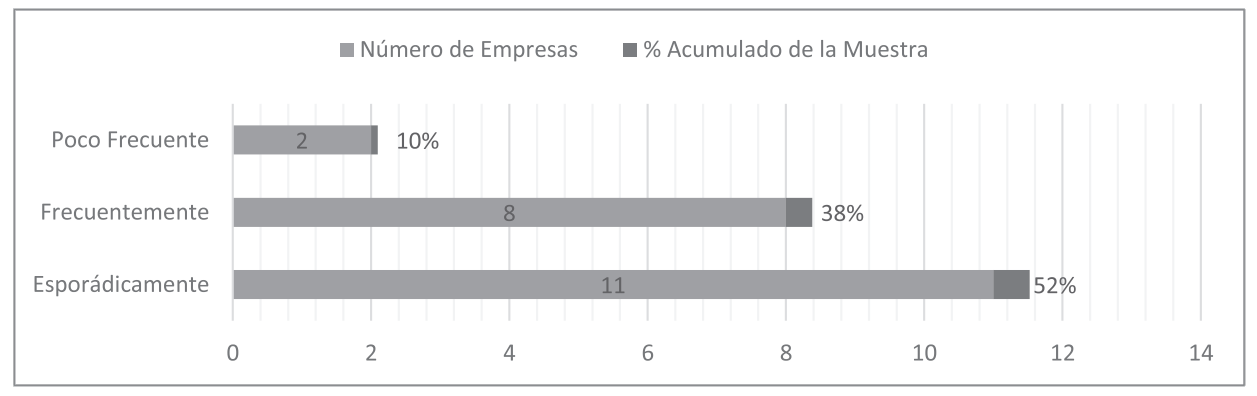

Figura 44. Clasificación de las empresas productoras según el tipo de producto elaborado.

Fuente: elaboración propia.

La producción en la cadena de suministro

A pesar de no tener una estructura sólida de planificación, se evidencia el cumplimiento de las órdenes de producción definidas por el cliente en un 95\%, infiriendo el compromiso en la ejecución de los valores acordados. Sin embargo, la relación existente entre las órdenes recibidas y la ejecución del proceso en función del tiempo, tienen una efectividad que muestra que el $5 \%$ la clasifica como baja permitiendo la aparición de faltantes en el cliente, el 24\% de las empresas manifiestan que la efectividad de ajuste es media baja representado en un $19 \%$, frente a un $48 \%$ que tienen buenos resultados de efectividad en el cumplimiento de las órdenes de materiales en función del tiempo. 
En cuanto a los indicadores del proceso productivo las empresas cuentan con estructuras definidas para el control estadístico al cual se le aplica controles periódicos. Sin embargo, existen oportunidades de fortalecimiento considerando que solo el $48 \%$ de las empresas los aplica. Dentro de los indicadores se evidencia el control de fallas en el proceso el cual es aplicado por el $71 \%$ de las empresas frente a un $29 \%$ que no los aplica. Cabe mencionar que el control de indicadores en esta etapa es fundamental considerando la incertidumbre asociada a deterioro del inventario. Las pérdidas por deterioro en el inventario de materia prima y producto terminado afectan los costos operacionales y la satisfacción del cliente. Las cifras obtenidas en cuando a la frecuencia de pérdidas por deterioro son considerablemente altas representadas en la Figura 46.

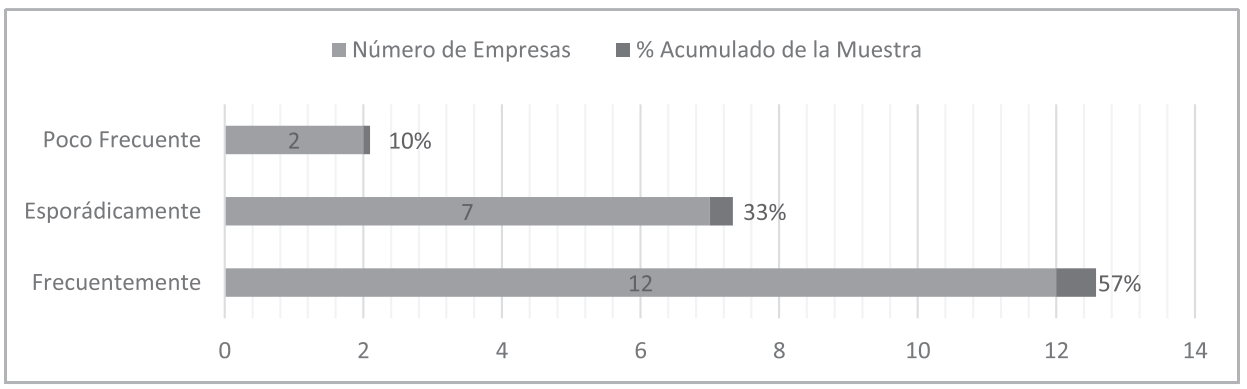

Figura 45. Frecuencia de inventario deteriorado en el fabricante.

Fuente: elaboración propia.

Las cifras de inventario deteriorado promueven la medición estadística en las empresas productoras a fin de establecer controles que reduzcan el riesgo. Es por lo que se determina otro de los estimulantes a la propuesta de producción de varios tipos de productos, debido a que, al ser la leche su principal materia prima, es también el producto que implica mayor riesgo por descomposición, teniendo una progresión en función del tiempo y temperatura. Dichas variables también intervienen en su proceso de transformación a los derivados. Los controles de pérdidas por deterioro son aplicados por el $62 \%$ de las empresas. El comportamiento de las variables mencionadas en la interacción con la leche implica la inversión de tecnología e infraestructura que permite la eliminación del riesgo por deterioro implicando a su vez una restricción de capacidad por almacenamiento. Esto puede justificar el grado de flexibilidad que el sector 
tiene para responder al mercado, la cual se midió en función del tiempo de respuesta obteniendo una valoración media baja en el 19\% de las empresas, una acentuación en la valoración media representando el $43 \%$, seguido del $33 \%$ la describe como media alta y finalizando con el 5\% de las empresas que cuentan con la infraestructura ideal para dar respuesta inmediata calificándola como alta.

Como se ha mencionado anteriormente la fuerza de trabajo es fundamental en el proceso productivo, sobre este aspecto la SC muestra que la fuerza de trabajo es limitada, donde el personal rota con frecuencia y es utilizado en el $81 \%$ de las empresas para desarrollar tareas múltiples dentro y fuera del área de producción, lo que hace que la mano de obra no sea especializada en función del trabajo.

\section{Distribución en la cadena de suministro}

La distribución se consideró en función de flujos en el interior de las organizaciones y flujo entre empresas, lo que permite determinar a nivel interno que existen muchas mejoras estructurales en cuando a flujos de procesos y compatibilidad de áreas. En cuanto al inventario puntualmente, no se realiza una distribución y asignación de posiciones considerando la rotación del inventario implicando que el $76 \%$ de las empresas esté incurriendo en flujos y recorridos innecesarios que generan el incremento de costos. Del mismo modo la incidencia de un proceso de distribución del inventario mal elaborado y fuerza de trabajo no especializada permite la generación de cruces de mercancía y devoluciones por parte del cliente considerando diferentes causales. En el índice de frecuencia de errores en despacho por cruce de materiales se obtuvo que el 29\% de empresas lo define como bajo al presentarse un gran número de errores en despacho, el $38 \%$ lo califica como medio bajo, mientras que el 33\% restante le otorga la calificación media representando alto índice de errores por despacho.

A fin de tener una idea de la facilidad de desarrollo del proceso de realización de una picking y destelle de pedidos, se obtiene que el 10\% de las empresas lo califica como media baja, el 43\% le otorga la calificación media, mientras que el 38\% medio alta al poder realizar la recogida de mercancías para consolidación de pedidas en forma ágil y rápida, finalizando con el $10 \%$ de empresas restantes que lo califican como alto manifestando tener las condiciones de espacio y organización adecuadas para el óptimo desarrollo. 
Se representa una cifra baja de errores en despacho. Esta situación es evidenciada en la frecuencia de ocurrencia de cruces en despacho teniendo que el 52\% de las empresas tiene cruces esporádicos, el 5\% es frecuente y el $43 \%$ restante es poco frecuente.

En cuanto a la distribución como flujo de materiales entre empresas, en la mayoría de los casos se realiza en vehículos tipo furgón que varían en capacidad de acuerdo con la necesidad del negocio y en casos particulares de empresas pequeñas se realizan en camionetas de estaca. Las labores son desarrolladas en un 57\% por operación propia, el 38\% utiliza un operador de distribución y el $5 \%$ restante comparte la actividad. Para todos los casos, a fin de medir grado de ocupación obteniendo que el 38\% de empresas califica la ocupación vehicular como baja, otro 5\% lo califica como medio bajo, esto quizás por vehículos demasiado grades adquiridos sin contemplar la demanda del mercado y la capacidad. Otro 10\% de empresas manifiestan tener un grado de ocupación medio y finalmente los porcentajes de empresa de 29\% y 19\% califican el grado de ocupación en media alta y alta. Esto permite determinar que las empresas están incurriendo en costo de oportunidad por subutilización de la capacidad vehicular en las empresas pequeñas. La Figura 47 orienta el porcentaje de ocupación vehicular estimado por las empresas.

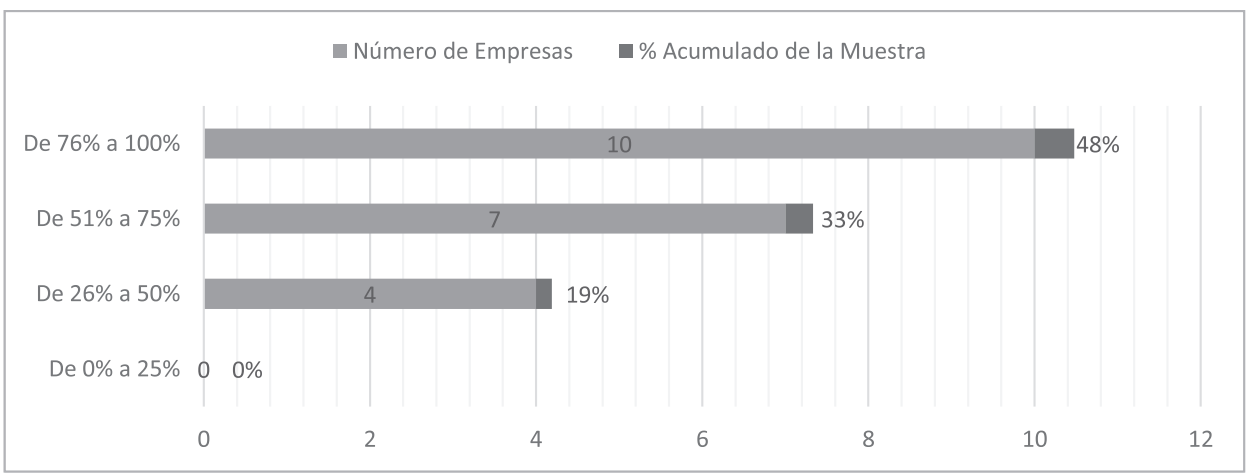

Figura 46. Porcentaje Estimado de Ocupación Vehicular .

Fuente: elaboración propia.

Considerando que las empresas de producción de queso en el Departamento de Sucre en su gran mayoría están clasificadas como pequeña empresa $86 \%$, la tendencia se ve reflejada en los canales comerciales utilizados para la comercialización de sus productos, evidenciando que el 
canal tradicional y directo son los de mayor cobertura. Teniendo que el canal tradicional mercado público tiene una utilización del $71 \%$ y tiendas del $81 \%$ mientras que el canal directo es utilizado en un $43 \%$. El resto de la cifra se sitúa en distribuidoras con $29 \%$ y grandes cadenas con $10 \%$. Cabe menciona que el canal distribución y grandes cadenas, cuenta con criterios de selección de productos a comercializar, considerando aspectos como la calidad, capacidad de respuesta del productor, precios, entre otros, que podría representar una limitante para su uso. Sin embargo, se puede apreciar que las empresas no limitan su comercialización a un solo canal, sino que hacen uso de varios de acuerdo con su interés y necesidad a fin de llegar a mayor número de clientes.

La medición de la flexibilidad en el proceso de distribución en función de cantidades disponibles para entregar en función del tiempo para hacer frente a la necesidad del cliente en cada uno de los canales comerciales mencionados, sustentan la importancia de definir canales de colaboración al presentar un comportamiento moderadamente flexible en el 33\% de las empresas y la rigidez de la relación fabricante - Cliente se evidencia en el $67 \%$ de empresas que manifiestan ser poco flexibles en la agilidad de respuesta. Estas cifras representan el bajo flujo de información en la cadena y la escasa colaboración en los niveles de inventario.

Finalmente se evidencia en el proceso de abastecimiento que solo el $24 \%$ de las empresas ha definido indicadores del control, reflejando que un $76 \%$ de las empresas no está controlando tiempos de operación, niveles de servicio, capacidad de respuesta, pedidos no entregado, devoluciones, entre otros.

Relaciones externas de la cadena de suministro

\section{Relación con proveedores}

Los proveedores de la cadena de suministro de lácteos del departamento de Sucre han orientado su relación de colaboración a contratos de descuentos por cantidad (95\%), definición de plazos de pago (52\%), acuerdos para compartir información (10\%) mostrando una relación básica de cantidades y costos.

El grado de efectividad de los canales de comunicación definidos en la cadena de suministro de acuerdo con la necesidad de cada empresa, muestran que el 5\% de las empresas manifiesta que es bajo, el 10\% lo 
define como moderadamente bajo, la mayor concentración de empresas lo describe en términos regulares representando un 48\%, seguido del 33\% que lo define como bueno mientras que el $5 \%$ de las empresas restantes manifiesta estar satisfecha con los canales propuestos de comunicación.

A pesar de que la colaboración entre los fabricantes y proveedores solo se limita a una relación costo y cantidad, se consultó sobre métodos de retroalimentación y de satisfacción con el proceso de abastecimiento representado a través de algún tipo de procedimiento de evaluación de desempeño y certificación de la labor, obteniendo que el 86\% de las empresas fabricantes - proveedores, no se preocupan por socializar las oportunidades de mejora en el proceso. Muy a pesar de que el 57\% de las empresas productoras manifiestan realizar reuniones periódicas con sus proveedores. Dentro de los aspectos a destacar en las reuniones, se extrae el $5 \%$ de las empresas las cuales definen objetivos conjuntos para alcanzar ventajas competitivas en el mercado.

Por su parte los proveedores de la cadena de suministros muestran un comportamiento rígido casi inflexible en cuanto a devoluciones de materiales por daños o excesos, representando el $24 \%$ de las empresas, mientras que un 10\% cede un poco ante la situación, por su parte el 49\% evalúa el grado de responsabilidad en la devolución y determina su participación, frente a un 19\% que acepta la devolución de los materiales para no afectar al productor. Sin embargo, cabe mencionar que en las visitas realizadas se evidenció que el proveedor de leche tiene la responsabilidad de transportar hasta las instalaciones del fabricante los litros de leche producidos o recogidos, los cuales antes de ingresar a los tanques de almacenamiento son revisados a través de pruebas fisicoquímicas para determinar el cumplimiento de las necesidades y especificaciones de la planta procesadora, en el caso de que la leche no cumpla esta es devuelta en su totalidad. Teniendo para ello una respuesta por parte del proveedor, que para el caso el 10\% de las empresas productoras manifestaron la terminación de la relación con el proveedor, teniendo este teniendo un tiempo de respuesta a la devolución deficiente, Por otro lado, el 38\% de las empresas productoras valoran el tiempo de respuesta relativamente bajo, mientras que el 33\% dan una calificación media a sus proveedores, otro $14 \%$ lo define como bueno y finalmente el 5\% restante tiene satisfacción total frente al tema. En la Figura 48 se observa la frecuencia de devoluciones. 


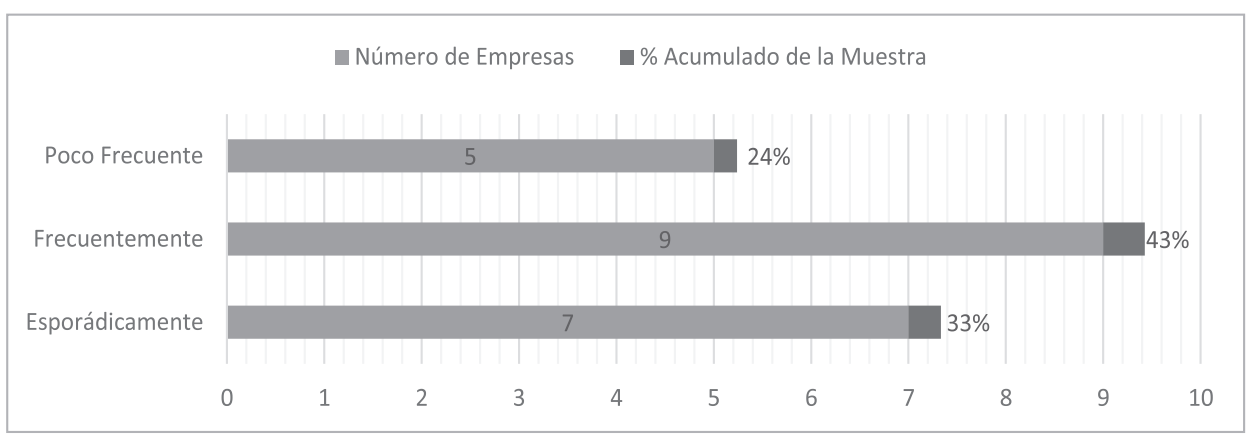

Figura 47. Frecuencia de devoluciones realizadas desde el productor al proveedor.

Fuente: elaboración propia.

Finalmente, el análisis de la relación entre fábricas y proveedores identifica que en esta etapa del proceso en ambas posiciones se definen indicadores de desempeño general y devoluciones en un 52\%, el $48 \%$ restante no cuenta con las estructuras definidas, pero efectúa control a través de otros mecanismos.

\section{Relación con clientes}

Por último, llegamos a la relación con el cliente, el cual es motor de todas las cadenas productivas del cual se extrae inicialmente el tipo de colaboración establecida en la cadena de suministro de queso en el Departamento de Sucre, identificando que se han definido descuentos por volumen aplicado por el 100\% de las empresas, plazos en los pagos 57\% y acuerdos para compartir información 14\%. Este último incluye información de demanda, niveles de inventario, bienes y otros servicios.

Para este caso, los canales de comunicación definidos para el funcionamiento de la relación son los tradicionales que incluyen correo electrónico, telefonía y contacto directo, teniendo una efectividad catalogada como excelente, sobresaliente, aceptable, insuficiente y deficiente, teniendo que el $5 \%$ es cociente y manifiesta que los canales definidos son insuficiente, el $71 \%$ los define como aceptable, mientras que el 24\% da el calificativo de sobre saliente al contar con otras herramientas tecnológicas como plataformas digitales, pagina web, entre otros. Sin embargo, el alcance de la relación no contempla en un 100\% la definición de procedimientos que 
permitan la retroalimentación en cuanto a la satisfacción de los productos y el proceso.

El contacto directo mencionado se efectúa a la hora de realización de reuniones periódicas desarrolladas por el 57\% de las empresas, donde intervienen aspectos conjuntos que permitan mejorar el proceso. Sin embargo, en las reuniones solo en el 10\% de las empresas se definen objetivos conjuntos para optimizar el rendimiento y generar ventajas competitivas.

El tema relacionado a devoluciones también se presenta en esta relación, evidenciando que el productor recibe el 100\% de los materiales deteriorados en el inventario del cliente siempre que este corresponda a vencimiento y que el volumen no comprometa la estabilidad de la empresa. La frecuencia estimada se presenta en la Figura 49, donde se evidencia que las devoluciones tienen una frecuencia alta.

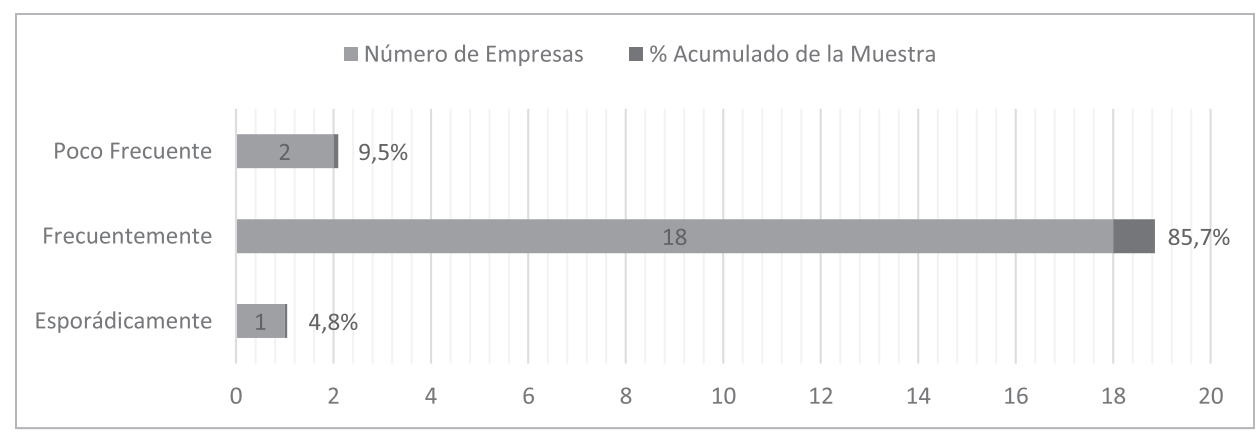

Figura 48. Frecuencia de Devoluciones por Parte del Cliente.

Fuente: elaboración propia.

Sin embargo, la situación cambia cuando el inventario pretende devolverse por exceso en el cliente dado que esta deriva netamente de una errónea planificación de la demanda. Para estos casos puntuales la empresas cuentan con diferentes grados de flexibilidad, identificando que el $5 \%$ de las empresas es totalmente rígida no permitiendo la devolución; el $10 \%$ presenta una flexibilidad baja que depende del tipo de cliente y motivo; por otro lado, y mostrando la mayor concentración de empresas en el término medio en el cual se evalúan las responsabilidades, así mismo el 24\% presenta una flexibilidad moderada, quedando un 5\% de empresas 
que aceptan todas las devoluciones con el fin de conservar la relación con el cliente, siendo posiblemente un escenario donde los volúmenes son relativamente pequeños.

Por otro lado, considerando la oportunidad de devoluciones por excesos de inventario, la valoración del tiempo de respuesta por parte de las empresas fabricantes varía en función del tamaño de la empresa, capacidad del vehículo y método de distribución. Dado que, en la mayoría de los casos, las devoluciones se hacen efectivas en la próxima entrega al cliente. Teniendo en cuenta esto, los tiempos de respuesta en el 5\% de la empresa son lentos, en el 19\% tiene un ritmo un poco más acelerado, un tiempo medio contiene el $52 \%$ de las empresas para finalmente el 24\% de empresas darle una respuesta casi inmediata.

Como estrategia de control se evidencia la definición de indicadores en el proceso de entrega y de devoluciones buscando garantizar la satisfacción del cliente y tener datos históricos por cada cliente específico. Teniendo como soporte de ello el 52\% de empresas las cuales han definido y aplican indicadores.

El análisis de los resultados permite la construcción de una estructura de mejores prácticas a la cadena de suministros de lácteos del departamento de Sucre, teniendo en cuenta los aspectos generales definidos en la caracterización. Se estima que las recomendaciones descritas en la Tabla 22, bien implementadas, representarían el crecimiento del sector e incremento de la competitividad a nivel local y nacional. 
Tabla 23. Estatura de mejores prácticas recomendadas a la cadena de suministro de lácteos del departamento de Sucre.

\begin{tabular}{|c|c|c|c|c|c|c|}
\hline \multicolumn{7}{|l|}{ Mejores Prácticas } \\
\hline \multirow[b]{2}{*}{ Argumento } & \multicolumn{6}{|c|}{ Aspectos de la cadena } \\
\hline & 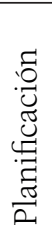 & 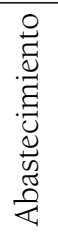 & 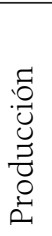 & 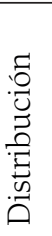 & 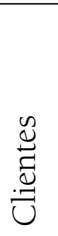 & 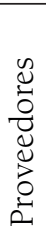 \\
\hline $\begin{array}{l}\text { Acuerdos de servicios conjuntos. Definir sistemas de } \\
\text { planificación de colaboración y suministro de } \\
\text { información. }\end{array}$ & & & & & $\mathrm{X}$ & $\mathrm{X}$ \\
\hline $\begin{array}{l}\text { Incluir aspectos ambientales en la gestión de } \\
\text { proveedores y compras. }\end{array}$ & & $X$ & & & & $\mathrm{X}$ \\
\hline Fortalecimiento del control estadístico de procesos. & $X$ & $X$ & $X$ & $X$ & $X$ & $X$ \\
\hline $\begin{array}{l}\text { Vincular el desempeño individual a los objetivos de } \\
\text { la organización, sus diferentes áreas de la } \\
\text { organización y la cadena de suministros. }\end{array}$ & $\mathrm{X}$ & & & & & \\
\hline $\begin{array}{l}\text { Realizar benchmarking de mejores prácticas con } \\
\text { otras empresas del sector. }\end{array}$ & $\mathrm{X}$ & & & & & \\
\hline Disponibilidad rápida de colaboradores. & & & $X$ & & & \\
\hline Capacitar continuas a los trabajadores. & $\mathrm{X}$ & & & & & \\
\hline $\begin{array}{l}\text { Planificación y colaboración de la capacidad } \\
\text { instalada de las empresas para mejorar la respuesta a } \\
\text { fluctuación de demanda. }\end{array}$ & $X$ & & & & & \\
\hline $\begin{array}{l}\text { Mejora en la clasificación de los trabajos con fuerza } \\
\text { de trabajo con funciones definida y especializada. }\end{array}$ & & & $\mathrm{X}$ & & & \\
\hline $\begin{array}{l}\text { Manufactura dividida en células de trabajo para } \\
\text { mayor flexibilidad. }\end{array}$ & & & $\mathrm{X}$ & & & \\
\hline $\begin{array}{l}\text { Plan preciso y aprobado de las instrucciones de } \\
\text { trabajo/procesos. }\end{array}$ & $X$ & & & & & \\
\hline $\begin{array}{l}\text { Control de desperdicios en procesos a través de } \\
\text { indicadores. }\end{array}$ & & $\mathrm{X}$ & $X$ & $\mathrm{X}$ & & \\
\hline
\end{tabular}




\begin{tabular}{|l|l|l|l|l|l|l|}
\hline $\begin{array}{l}\text { Utilización de sistemas informáticos para mejora del } \\
\text { flujo de información en procesos internos y } \\
\text { externos. }\end{array}$ & & $\mathrm{X}$ & $\mathrm{X}$ & $\mathrm{X}$ & $\mathrm{X}$ & $\mathrm{X}$ \\
\hline Socialización de resultados de rendimiento. & & $\mathrm{X}$ & $\mathrm{X}$ & $\mathrm{X}$ & $\mathrm{X}$ & $\mathrm{X}$ \\
\hline Control de los niveles de inventario ajustado. & & $\mathrm{X}$ & $\mathrm{X}$ & & $\mathrm{X}$ & $\mathrm{X}$ \\
\hline $\begin{array}{l}\text { Mejoras en la distribución del Layout de planta e } \\
\text { inventario. }\end{array}$ & $\mathrm{X}$ & $\mathrm{X}$ & & & \\
\hline Ubicación de planta en relación socios en la cadena. & & & & & $\mathrm{X}$ & $\mathrm{X}$ \\
\hline $\begin{array}{l}\text { Control de calidad procesos para garantizar la } \\
\text { conformidad de los productos. }\end{array}$ & & $\mathrm{X}$ & $\mathrm{X}$ & $\mathrm{X}$ & & \\
\hline Mejoras en devoluciones y plazos de recogida. & & & $\mathrm{X}$ & & $\mathrm{X}$ & $\mathrm{X}$ \\
\hline $\begin{array}{l}\text { Diseño de productos, servicios y procesos en } \\
\text { colaboración con: }\end{array}$ & & & & & $\mathrm{X}$ & $\mathrm{X}$ \\
\hline Reabastecimiento rápido y efectivo. & & $\mathrm{X}$ & & & $\mathrm{X}$ & \\
\hline $\begin{array}{l}\text { Colaboración en la gestión de inventarios que } \\
\text { garantice los niveles óptimos y la respuesta rápida y } \\
\text { efectiva al consumidor. }\end{array}$ & & & $\mathrm{X}$ & & $\mathrm{X}$ & $\mathrm{X}$ \\
\hline $\begin{array}{l}\text { Ventas directas en el fortalecimiento de la relación } \\
\text { con el cliente final. }\end{array}$ & & & & & $\mathrm{X}$ & \\
\hline
\end{tabular}

\section{Conclusiones y recomendaciones}

Fuente: elaboración propia.

Los resultados obtenidos en el estadio de caracterización reflejan la necesidad de fortalecimiento del sector lácteo del departamento de Sucre, reflejando mejorar su estructura de planificación general de operaciones que permita determinar sus necesidades internas y externas, estableciendo controles en la ejecución y medición del beneficio. Dentro de las limitantes identificadas para el desarrollo de procesos colaborativos se identifica la cultura de la región, que se evidencia rígida, con flujo de información limitado asociado a la desconfianza entre integrantes de la cadena. Lo cual también se vio reflejado a la hora de recolectar la información del estudio.

Para el fortalecimiento del sector, la colaboración juega un papel importante puesto que este tipo de relaciones dentro de los eslabones de la cadena puede conciliar los intereses de los distintos actores y lograr una 
planificación de la producción, creación de valor, relaciones mutuamente beneficiosas con los proveedores y clientes, minimización de costos, incrementar los niveles tecnológicos, cumplir con la demanda del mercado y acceder a otros segmentos de mercado, reflejándose en valor agregado para el cliente y ventajas comparativas que potencialicen la competitividad de la cadena y una equitativa distribución de los márgenes de ganancia.

En general la cadena de suministro tiene un tiempo relativamente corto en el mercado, reflejando una relación directamente proporcional a su tamaño. Sus esquemas colaborativos son casi inexistentes y limitados a suministros y cantidades sin ningún tipo de esquema de apoyo, suministro de datos, etc. Las características identificadas en la cadena destacan la individualidad en los procesos y las limitantes establecidas por la cultura de la región en cuanto al suministro de información.

En cuanto al personal, en la cadena se identifica una mano de obra poco calificada y cualificada, con estructuras de formación muy limitadas. Existiendo una gran rotación de la mano de obra, la cual no es estática ni específica para cada función, representando ventaja aparente en cuanto al escaso truncamiento de los procesos, pero que impacta en el tiempo de ejecución de los procesos. Los errores en el flujo aguas arriba y aguas abajo son constantes, en gran parte representados por fallas en procesos y la otra parte asociada a el grado de deterioro de los productos.

Los controles estadísticos son unos de los aspectos a intervenir por la cadena, no contando con estructuras sólidas definidas para evaluar y determinar el desempeño de los procesos. Así mismo, es necesario desarrollar capacitaciones permanentes en relación con los procesos de obtención, recolección, manipulación y transporte de leche cruda involucrando los actores, empresas de la región, entidades del estado, agremiaciones y demás participantes de la cadena. 


\section{Referencias Bibliográficas}

Aguilera-Díaz, M. M. (2005). La economía del departamento de Sucre: ganadería y sector público. . Documentos de Trabajo Sobre Economía Regional y Urbana, No. 63.

Batt, P. J., \& Purchase, S. (2004). Managing collaboration within networks and relationships. Industrial marketing management, 33(3), 169-174.

Berna, L. (2011). Modelo de ruteo para entregas de mercancías a clientes por terceros en Sector Retail.

Betts, T., \& Tadisina, S. K. (2009). Supply chain agility, collaboration, and performance: how do they relate? , 1-22. POMS 20th Annual Conference, 1-22.

Bititci, U. S., \& Mokadam, M. (2010). Development of a collaborative supply chain model. In 17th International Conference of the European Operations Management Association, 1-10.

Bohorquez, N., Buitrago, A., Joya, M., Montaña, X., \& Rivera, H. (2012). Análisis Estructural de Sectores Estratégicos: Sector productos Lácteos. (Documento de Investigación; 135). Bogotá: Editorial Universidad del Rosario.

Bowersox, D. J., Closs, D. J., \& Stank, T. P. (2003.). How to master cross-enterprise collaboration. Supply Chain Management Review, SUPPLY CHAIN MANAGEMENT REVIEW, 7(4), 18-27.

Calderòn, M. E., \& Orjuela, J. A. (2004). Competitividad en la Cadena Agroindustrial láctea. Universidad Distrital Francisco José de Caldas, Bogotà, CO, No. 338.177 Cl46c.

Cao, M., Vonderembse, M. A., Zhang, Q., \& Ragu-Nathan, T. S. (2010). Supply chain collaboration: conceptualisation and instrument development. International Journal of Production Research, 48(22), 6613-6635.

Castillo, H., \& Bermeo, J. (2013). Propuestas de Sistemas Inteligentes para la Implementación de un Modelo de Gestión Triple A en la Cadena de Suministros de Centros de Acopio de Leche cruda en la Provincia de Azuay. Cuenca - Ecuador: Universidad Plitecnica Salesiana.

Celis, M., \& Juárez, D. (2009). Microbiología de la Leche. Editorial de la Universidad Tecnológica Nacional - edUTecNe.

Chauhan, S., Eremeev, A., Romanova, A., Servakh, V., \& Woeginger, G. (2005). Approximation of the supply sheduling problem. Operational Reseach Letters $\mathrm{N}^{\circ} 33,249-254$. 
Chen, L. X., \& Li, J. (2008). Retailer's order policy for perishable products with demand information updating. Mathematics in Practice and Theory, 1, pp. 34-40.

Chen, W., Li, J., \& Jin, X. (15 de April de 2016). The replenishment policy of agri-products with stochastic demand in integrated agricultural supply chains. Expert Systems with Applications, 48, 55-66.

Cho, M., Song, M., Comuzzi, M., \& Yoo, S. (2017). Evaluating the effect of best practices for business process redesign: An evidence-based approach based on process mining techniques. Decision Support Systems, 104, 92-103.

Chounta, I. A., Hecking, T., Hoppe, H. U., \& Avouris, N. (2014). Two Make a Network: Using Graphs to Assess the Quality of Collaboration of Dyads. Collaboration and Technology. Springer, 53-66.

Comité Nacional Sistema Producto Bovino Leche. (2015). Situation de la Lechería a Nivel Mundial. Mexico: US Dairy Export Council.

CONPES. (2010). 3675. Política Nacional para Mejorar la Competitivad del Sector Lácteo Colombiano. Bogotá: Consejo Nacional de Política Económica y Social. Departamento Nacional de Planeación.

Consejo Nacional Lácteo. (2011). Propuesta de Valor Cadena Láctea Colombiana. Programa de Transformación Productiva (PTP). Bogotá.

DANE. (2012). Informe de Coyuntura Ecónomica Regional Departamento de Sucre. Sincelejo: Banco de la República.

DANE. (06 de Noviembre de 2015). Tercer Censo Nacional Agropecuario. Décima entrega resultados 2014- cifras preliminares. Bogotá, Colombia: Departamento Administrativo Nacional de Estadística.

Dania, W. A., Xing, K., \& Amer, Y. (2018). Collaboration behavioural factors for sustainable agri-food supply chains: A systematic review. Journal of Cleaner Production, 186, 851-864.

Daugherty, P. J., Richey, R. G., Roath, A. S., \& Genchev, S. E. (2006). Is Collaboration Paying Off for Firms? Business Horizons, 49(1), 61-70.

Departamento Nacional de Planeación. (2007). Agenda Interna para la Productividad y la Competitividad de Sucre. Bogotá.

Espinosa , F. A. (2012). TLC y Carne de Bovino. Carta FEDEGAN. Federación Colombiana de Ganaderos, 142-146.

Estrada, R., \& Holmann, F. (2008). Competitividad de la Producción de Leche frente a los Tratados de Libre Comercio en Nicaragua, Costa Rica y Co- 
lombia. (Documento de Trabajo no. 207). Cali: Co: Centro Internacionald Agricultura Tropical (CIAT), International Livestock Research Institute (ILRI).

FAO \& FEPALE. (2012). Situación de la Lechería en América Latina y el Caribe en 2011, Observatorio de la Cadena Lechera. Ofcina Regional de la FAO para América Latina y el Caribe, División de Producción y Sanidad Animal. Chile.

Faust, V., Christens, B. D., Sparks, S. M., \& Hilgendorf, A. E. (2015). Exploring relationships among organizational capacity, collaboration, and network change. Psychosocial Intervention, 24(3), 125-131.

Fawcett, S. E., Magnan, G. M., \& McCarter, M. W. (2008). Benefits, barriers, and bridges to effective supply chain management. Supply Chain Management: An International Journal, 13(1), 35-48.

FEDEGAN. (2009). Lo que usted necesita saber sobre la leche en Colombia. Bogotá.

FEDEGAN. (2012). Retos de la Globalozación en el Mercado lácteo. Bogotá: Federación Nacional de Ganaderos.

FEDEGAN. (2013). Proyecto de Ley para el sector Lácteo: ¿Qué piden los ganaderos al Gobierno? Carta No. 135, 14-33. Bogotá.

FEDEGAN. (2015). Informe Especial: Importancia de Modernizar las Lecherías. Carta FEDEGAN - Federación Colombiana de Ganaderos, 151, 12-21.

Fisher, M., Hammond, J., Obermeyer, W., \& Raman, A. (1997). Configuring a supply chain to reduce the cost of demand uncertainty. Production and operations management, 6(3), 21l-225.

Fluck, C. (2014). Closing the Gap between the Current and Potential Conditions in the Cocoa (Production) Industry: Comparing Colombia and Mexico. Bachelor`s Thesis, University of Twente, 1-37.

Francois, J., Moad, K., Bourrieres, J. P., \& Lebel, L. (2017). A tactical planning model for collaborative timber transport. IFAC-PapersOnLine, 50(1), 11713-11718.

FUNDACIÓN ALPINA. (2012). Análisis de Mercado. Popayán (Colombia): Aalcaldia municipal de Popayán Y Universidad el Cauca.

Fundesarrollo. (Junio de 2014). La Región Caribe en Cifras. Obtenido de Fundación para el Desarrollo de Caribe: http://www.fundesarrollo.org.co/ wp-content/uploads/2014/06/Regi\%C3\%B3n-Caribe-en-Cifras.pdf 
FUNDESARROLLO. (2014). La Región Caribe en cifras. Fundación para el Desarrollo del Caribe.

Gligor, D. M., \& Holcomb, M. (2013). The role of personal relationships in supply chains: an exploration of buyers and suppliers of logistics services. International Journal of Logistics Management, 24(3), 328-355.

Govindan, K., Soleimani, H., \& Kannan, D. (2014). Reverse logistics and closed-loop supply chain: A comprehensive review to explore the future. European Journal of Operational Researh, 1-57.

Govindan, K., Soleimani, H., \& Kannan, D. (2014). Reverse logistics and closed-loop supply chain: A comprehensive review to explore the future. European Journal of Operational Research, 1 - 57.

Grocery Manufacturers Asocciations. (2005). Manual de la Cadena de Abastecimiento de Productos Alimenticios. Washinton.

Guzman, K. (2013). La industria láctea en Valledupar: primera en la región Caribe [online]. Recuperado el Agosto de 2015, de http://www.banrep.gov.co/docum/Lectura_finanzas/pdf/dtser_184.pdf/

Han , J. H., Wang, Y., \& Naim, M. (2017). Reconceptualization of information technology flexibility for supply chain management: An empirical study. International Journal of production Econimics, 187, 196-215.

Heirati, N., \& Siahtiri, V. (2017). Heirati, N., \& Siahtiri, V. (2017). Driving service innovativeness via collaboration with customers and suppliers: Evidence from business-to-business services. Industrial Marketing Management, .

Hernández , F., Vertel, M., \& Porto, I. (2016). Análisis Factorial Múltiple de la competitividad de empresas lácteas del municipio de Sincelejo, Colombia. Agronomía Colombiana, 34(1Supl), S36-S40.

Hernández, F. D., Bustamante, L. F., \& Porto, I. A. (2014). Evaluación del grado de competitividad de cinco empresas del sector lechero de Sincelejo, Colombia. Revista de Investigación, Desarrollo e Innovación, 5(1), 8-19.

Howeg, M., Disney, S., Holmstrom, J., \& Smaros, J. (2005). Supply chain collaboration:: Making sense of the strategy continuum. European management journal, 23(2), 170-181.

Instituto Colombiano Agropecuario - ICA. (2016). Censo Pecuario Nacional. Bogotá: Ministerio de Agricultura. 
Jaramillo, A., \& Areiza, A. (2012). Market Analysis of Milk and Dairy Products in Colombia (2008-2012). Bogotá: Super Intendencia de Industria y Comercio.

Jiménez, J., \&\& Hernández, S. (2002). Marco conceptual de la cadena de suministro: un nuevo enfoque logístico. En S. d. Transportes, Publicación Técnica No. 215 (págs. 1-272). Sanfandila, Qro: Instituto Mexicano del Transporte.

Jonkman, J., Bloemhof, J. M., Van der Vorst, J. G., \& Van der Padt, A. (2017). Selecting food process designs from a supply chain perspective. Journal of Food Engineering, 195, 52-60.

Kalwani, M. U., \& Narayandas, N. (1995). Long-term manufacturer-supplier relationships: do they pay off for supplier firms? The Journal of marketing, 1-6.

Kelly, A., Leitner, G., \& Merin, U. (2011). Milk quality and udder health: Test Methods and Standards. Encyclopedia of Dairy Sciences. 2 ed. San Diego (USA), 894 - 901.

Kohli, A. S., \& Jensen, J. B. (2010). Assessing effectiveness of supply chain collaboration: an empirical study. Supply chain forum: An international journal, $11(2), 2-16$.

Kotabe, M., Martin, X., \& Domoto, H. (2003). Gaining from vertical partnerships: knowledge transfer, relationship duration, and supplier performance improvement in the US and Japanese automotive industries. Strategic Management Journal, 24(4), 293-316.

Kumar, G., Banerjee, R. N., Meena, P. L., \& Ganguly, K. K. (2017). Joint planning and problem solving roles in supply chain collaboration. IIMB management review, 29(1), 45-57.

Lee, H. L., Padmanabhan, V., \& Whang, S. (1997). Information distortion in a supply chain: The bullwhip effect. Management Science, 43(4), 546-558.

Lee, H., Kim, M. S., \& Kim, K. K. (2014). Interorganizational information systems visibility and supply chain performance. International Journal of Information Management, 34(2), 285-295.

Li, D., Wang, X., Chan , H., \& Manzini, R. (2014). Sustainable Food Supply Chain Management. International Journal of Production Economics, 152, 1-8.

Liao, S. H.,Hu, D. C., \& Ding, L. W. (2017). Assessing the Influence of Supply Chain Collaboration Value Innovation, Supply Chain Capability and Competitive Advantage in Taiwan's Networking Communication Industry. International Journal o Production Economics, 191, 143-153. 
Liu, C., \& Yao, J. (2018). Dynamic supply chain integration optimization in service mass customization. Computers \& Industrial Engineering, 120, 42-52.

Lombana, J., Martinez, D., Valverde, M., Rubio, J., Castrillón, J., \& Marino, W. (2012). Caracterización del Sector Ganadero del Caribe Colombiano. 1-76.

Martínez, M., \&\& Gómez, C. (2013). Compositional and Hygienic Quality of Raw Molk Received in Dairies in Sucre, Colombia. Biotecnología en el Sector Agropecuario y Agroindustrial. Vol 11 No. 2, 93-100.

Martínez, M., \&\& Gómez, C. (2013). Compositional and Hygienic Quality of Raw Molk Received in Dairies in Sucre, Colombia. Biotecnología en el Sector Agropecuario y Agroindustrial. Vol ll No. 2, 93- 100.

Martìnez, M., Serpa, J., \& Gomez, C. (2009). Diagnostico de la calidad Composicional e Higiénico sanitarias de la Leche Cruda en Centros de Acopio y Plantas Procesadoras del Departamento de Sucre. Hipertexto Ltda. Sincelejo, Colombia, 1-10.

Mathuramaytha, C. (2011). Supply chain collaboration-What's an outcome? A theoretical model. International Conference on Financial Management and Economics IPEDR, IACSIT Press, Singapore, 11, 102-108.

Meneses, R., Estrada, D., Chantré, C., \& López, F. (2015). Characterization in the non formal chain of raw milk at the municipality of Popayan. Biotecnología en el Sector Agropecuario y Agroindustrial Vol 13 No. 2 , 130-139.

Miemczyk, J., Johnsen, T., \& Macquet, M. (2012). Sustainable purchasing and supply management: a structured literature review of definitions and measures at the dyad, chain and network levels. Supply Chain Management International Journal, 17(5), 478-496.

Ministerio de transporte. (2010). Plan nacional de desarrollo 2010 - 2014. Sector transporte. Bogotá: ministerio de transporte.

Ministerio de Agricultura y Desarrollo Rural. (2005). La Cadena de lácteos en Colombia. Una mirada global de su estructura y dinámica 1995-2005. Bogotá, Marzo de 2005: Observatorio Agrocadenas de Colombia.

Ministerio de Agricultura y Desarrollo Rural. (2007). Agenda prospectiva de Investigación y Desarrollo Técnológico para la Cadena Láctea Colombiana. Bogotá D.C: Giro Editores Ltda.

Ministerio de Agricultura y Desarrollo Rural. (2010). Dirección de Política Sectorial - Grupo de Análisis Sectorial. Boletín de análisis por producto. boletín No. 6. Bogotá: MADR. 
Ministerio de Trabajo. (2014). Plan Departamental de Empleo de Sucre. Convenio № 188. Programa de Asistencia Técnica para el Fortalecimiento de las Políticas de Empleo, Emprendimiento y Generación de Ingresos. Fundación Panamericana para el Desarrollo - FUPAD. Opciones Gráficas Editores Ltda.

Ministrerio de Salud y Protección Social. (2011). Decreto Número 1880 de 2011. Por el cual se señalan los requisitos para la comercialización de leche cruda para consumo humano directo en el territorio nacional. Bogotá.

Moghaddam, M., \& Nof, S. Y. (2016). Real-time optimization and control mechanisms for collaborative demand and capacity sharing. International Journal of Production Economics, 171(4), 495-506.

Mojica, F., Trujillo, R., Castellanos, D., \& Bernal, N. (2007). Agenda prospectiva de investigación y desarrollo tecnológico de la cadena láctea colombiana. Bogotá: Ministerio de Agricultura y Desarrollo Rural. Obtenido de www. minagricultura.gov.co.

Murphy, M., O’Mahony, M., Shalloo, L., French, P., \& Upton, J. (2014). Comparison of modelling techniques for millk-production forecasting. Journal of Dairy Science, 97, 3352-3363.

Olivero, R., Aguas, Y., \& Cury, K. (2011). Comercialización de Leche Cruda en Sincelejo, Sucre, Colombia. Revista Colombiana de Ciencia Animal-RECIA, 3(1), 157-163.

Olivero, R., Aguas, Y., \& Cury, K. (2011). Raw Milk Marketing in Sincelejo, Sucre, Colombia. Rev. Colombiana cienc. Anim. 3(1), 157- 163.

Orjuela, A. (2013). Estudio de la Cadena Láctea y su asporte a la Competitividad de la zona noroccidental del Municipio de Pasto, Departamento de Nariño. San Juan de Pasto.

Perny, J. F., Crump, P. M., Hernandez, L. L., \& Reinemann, D. J. (2018). Association of milking interval and milk production rate in an automatic milking system. Journal of Dairy Science, 101, 1616-1625.

PROEXPORT. (2011). Sector Lácteo en Colombia. Bogotá, D.C.: PROEXPORT COLOMBIA.

Proexport. (2013). Sucre Aprovecha los TLC. Revista de las Oportunidades Proexport Colombia, 1-24.

Red Nacional de Agencias de Desarrollo Local. (2013). Plan Estrategico Departamental de Ciencia, Tecnología e Innovación de Sucre. Sucre Innova, Sucre 
Transforma - PEDCTI. Convenio 0592-2012. Sincelejo: Gobernación de Sucre.

Red Nacional de Agencias de Desarrollo Local. (2013). Plan Estrategico Departamental de Ciencia, Tecnología e Innovación de Sucre. Sucre Innova, Sucre Transforma. Convenio 0592-2012. Sincelejo: Gobernación de Sucre.

Sana, S. S. (2012). A collaborating inventory model in a supply chain. Economic Modelling, 29(5), 2016-2023.

Secretaria de Desarrollo Económico y Medio Ambiente. (2016). Informe de Coyuntura Departamental. Sincelejo: Gobernación de Sucre.

Shortall, J., Foley, C., Sleator, R. D., \& O'Brien, B. (2018). The effect of dairy cow breed on milk production, cow traffic and milking characteristics in a pasture-based automatic milking system. Livestock Science, 209, 1-7.

Simatupang, T. M., \& Sridharan, R. (2005). The collaboration index: a measure for supply chain collaboration. International Journal of Physical Distribution \& Logistics Management, 35(1), 44-62.

Singh, P. J., \& Power, D. (2009). The nature and effectiveness of collaboration between firms, their customers and suppliers: a supply chain perspective. Supply Chain Management: An International Journal, 14(3), 189-200.

Tsanos, S., Zografos, G. K., \& Harrison, A. (2014). Developing a conceptual model for examining the supply chain relationships between behavioural antecedents of collaboration, integration and performance. The International Journal of Logistics Management, 25(3), 418-462.

Weber, C. A., Current, J., \& Desai, A. (2000). An optimization approach to determining the number of vendors to employ. Supply Chain management: An international journal, 5(2), 90-98.

Wu, I. L., \&\& Chiu, M. L. (2018). Examining supply chain collaboration with determinants and performance impact: Social capital, justice, and technology use perspectives. International Journal of Information Management, 39, 5-19.

Zhang, F., Murphy, M., Shallo, L., Ruelle, E., \& Upton, J. (2016). An automatic model configuration and optimization system for milk production forecasting. Computers and Electronics in Agriculture, 100-11l.

Zhang, Q., \& Cao, M. (2018). Exploring antecedents of supply chain collaboration: Effects of culture and interorganizational system appropriation. International Journal of Production Economics, 195, 146-157. 